\title{
RICHARDSON EXTRAPOLATION AND DEFECT CORRECTION OF MIXED FINITE ELEMENT METHODS FOR ELLIPTIC OPTIMAL CONTROL PROBLEMS
}

\author{
Yanping Chen, Yunqing Huang, and Tianliang Hou
}

\begin{abstract}
In this paper asymptotic error expansions for mixed finite element approximations to a class of second order elliptic optimal control problems are derived under rectangular meshes, and the Richardson extrapolation of two different schemes and interpolation defect correction can be applied to increase the accuracy of the approximations. As a byproduct, we illustrate that all the approximations of higher accuracy can be used to form a class of a posteriori error estimators of the mixed finite element method for optimal control problems.
\end{abstract}

\section{Introduction}

The aim of this paper is to discuss the asymptotic behavior of the mixed finite element approximation for a elliptic optimal control problem described as follows:

$$
\min _{u \in K \subset U}\left\{\frac{1}{2}\left\|y-y_{d}\right\|_{W}^{2}+\frac{1}{2}\|u\|_{U}^{2}\right\}
$$

subject to the state equation

$$
-\operatorname{div}(A \nabla y)+c y=f+B u, \quad x \in \Omega
$$

with the boundary condition

$$
A \nabla y \cdot \boldsymbol{n}=0, \quad x \in \partial \Omega,
$$

where $\Omega \subset \mathbb{R}^{2}$ is a bounded and convex open set with the Lipschitz boundary $\partial \Omega, \boldsymbol{n}$ indicates the outward unit normal vector along $\partial \Omega, L^{2}(\Omega)$ stands for

Received July 7, 2010; Revised May 24, 2011.

2010 Mathematics Subject Classification. Primary 35J15, 65K10.

Key words and phrases. optimal control problems, mixed finite element methods, asymptotic expansions, interpolation postprocessing, defect correction, a posteriori error estimators.

The first author is supported by the Foundation for Talent Introduction of Guangdong Provincial University, Guangdong Province Universities and Colleges Pearl River Scholar Funded Scheme (2008) and National Science Foundation of China (10971074). The second author is supported in part by the NSFC Key Project(11031006). The third author is supported by Hunan Provincial Innovation Foundation for Postgraduate CX2010B247. 
the usual $L^{2}$-inner product space, $K$ is a nonempty closed convex set in $L^{2}(\Omega)$, $f, y_{d} \in L^{2}(\Omega), B$ is a continuous linear operator from $U=L^{2}(\Omega)$ to $L^{2}(\Omega)$, $W=L^{2}(\Omega) . \quad c(x) \in L^{\infty}(\Omega)$ and there exists a constant $c_{*}>0$ such that $c(x) \geq c_{*}, \forall x \in \Omega$. The coefficient matrix $A \in L^{\infty}\left(\Omega ; \mathbb{R}^{2 \times 2}\right)$ is a symmetric and uniformly elliptic, i.e., $A(x)$ is a symmetric and positive definite $2 \times 2$ matrix and there exists a constant $c_{\star}>0$ satisfying for any vector $X \in \mathbb{R}^{2}$, $X^{T} A(x) X \geq c_{\star}\|X\|^{2}$ for almost all $x \in \Omega$.

Optimal control problems [27] have been extensively utilized in many aspects of the modern life such as social, economic, scientific and engineering numerical simulation. Due to the wide applications of these problems, they must be solved successfully with efficient numerical methods. Among these numerical methods, finite element method is a valid numerical method of studying the partial differential equation, but it is not deeply studied in solving optimal control problems. There have been many studies on this aspect, see $[33,19,20,30,12,3,16,28,1,2]$. A systematic introduction of finite element method for PDEs and optimal control can be found in, for example, $[32,31,17]$. In the recent years, we utilized the mixed finite element method to solve the optimal control problems. In $[7,8,9]$, we obtained the error estimates and superconvergence of mixed finite element methods for elliptic optimal control problems. In [10], we derived a posteriori error estimates of mixed finite element methods for elliptic optimal control problems. We also investigated the parabolic optimal control problems by mixed finite element methods, see $[36,11]$. Very recently, in [29], in order to increase the accuracy of finite element approximations for optimal control problems, they studied two numerical approaches of higher accuracy, namely, the Richardson extrapolation schemes and an interpolation defect correction method.

It is well known that the extrapolation method, which was established by Richardson in 1926, is an efficient procedure for increasing the accuracy of approximation of many problems in numerical analysis. The effectiveness of this technique relies heavily on the existence of an asymptotic expansion for the error. This technique has been well demonstrated in its applications to the finite element and the mixed finite element methods for elliptic partial differential equations [4, 6, 22, 34, 35], parabolic partial differential equations [18], integral and integro-differential equations $[22,24,25,26,38]$, and to the boundary element methods and collocation methods in [37] and [21], respectively. The defect correction (Galerkin and Petrov-Galerkin) finite element by means of an interpolation postprocessing technique is another numerical method to obtain approximations of higher accuracy, which has been proved for a wide variety of models, see, for example, [22, 23, 5], and the references cited therein.

Our objective in this paper is to present an analysis for the Richardson extrapolation method of two different forms and an interpolation defect correction method for the mixed finite element approximations in the $L^{2}$-norm. Firstly, we derive the asymptotic expansion of the error in the mixed finite element solution, by the asymptotic expansion the Richardson extrapolation 
of two different types and the interpolation defect correction can be applied to generate mixed finite element approximations of higher accuracy. In addition, by means of these approximations with higher accuracy, a class of a posteriori estimators are constructed for this mixed finite element method.

The paper is organized as follows: In Section 2, the approximation subspace and the variational formula of (1)-(3). Also, the asymptotic expansion for the Raviart-Thomas projection is presented for the future need. In Section 3, we investigate the asymptotic expansion of the error between the mixed finite element solution and the Raviart-Thomas projection of the exact solution to the model problem in the $L^{2}$-norm. Section 4 deals with an interpolation defect correction approximation in the $L^{2}$-norm based on the results given in Section 3. Furthermore, at each end of Sections 3 and 4, a posteriori error estimators are furnished as by-products of these numerical solutions with higher convergence rates. In the last section, we briefly give conclusions and some possible future work.

We adopt the standard notation $W^{m, p}(\Omega)$ for Sobolev spaces on $\Omega$ with a norm $\|\cdot\|_{m, p}$ given by $\|v\|_{m, p}^{p}=\sum_{|\alpha| \leq m}\left\|D^{\alpha} v\right\|_{L^{p}(\Omega)}^{p}$, a semi-norm $|\cdot|_{m, p}$ given by $|v|_{m, p}^{p}=\sum_{|\alpha|=m}\left\|D^{\alpha} v\right\|_{L^{p}(\Omega)}^{p}$. We set $W_{0}^{m, p}(\Omega)=\left\{v \in W^{m, p}(\Omega)\right.$ : $\left.\left.v\right|_{\partial \Omega}=0\right\}$. For $p=2$, we denote $H^{m}(\Omega)=W^{m, 2}(\Omega), H_{0}^{m}(\Omega)=W_{0}^{m, 2}(\Omega)$, and $\|\cdot\|_{m}=\|\cdot\|_{m, 2},\|\cdot\|=\|\cdot\|_{0,2}$. In addition $C$ or $c$ denotes a general positive constant independent of $h$.

\section{The asymptotic expansion}

In this section we give the weak variational formula and the mixed finite element method for the elliptic optimal control problem (1)-(3). For the sake of simplicity of analysis, we take the domain $\Omega$ to be a rectangle and $B=I$ in this paper.

Next, we introduce the co-state elliptic equation

$$
-\operatorname{div}(A \nabla z)+c z=y-y_{d}, \quad x \in \Omega
$$

with the boundary condition

$$
A \nabla z \cdot \boldsymbol{n}=0, \quad x \in \partial \Omega .
$$

Let

$$
\boldsymbol{V}=H(\operatorname{div} ; \Omega)=\left\{\boldsymbol{v} \in\left(L^{2}(\Omega)\right)^{2}, \operatorname{div} \boldsymbol{v} \in L^{2}(\Omega)\right\}
$$

equipped with the following norm given by

$$
\|\boldsymbol{v}\|_{\mathbf{v}}=\|\boldsymbol{v}\|_{H(\operatorname{div} ; \Omega)}=\left(\|\boldsymbol{v}\|_{0, \Omega}^{2}+\|\operatorname{div} \boldsymbol{v}\|_{0, \Omega}^{2}\right)^{1 / 2} .
$$

In addition, set

$$
\mathbf{V}_{0}=\{\mathbf{v} \in \mathbf{V}: \mathbf{v} \cdot \boldsymbol{n}=0, \mathbf{x} \in \partial \Omega\}
$$

To consider the mixed finite element approximation of our convex optimal control problems, we need a weak form of the optimal control problem (1)-(3). 
We recast (1)-(3) in the following weak form: (CCP) find $(\boldsymbol{p}, y, u) \in \boldsymbol{V}_{0} \times W \times U$ such that

$$
\begin{aligned}
& \min _{u \in K \subset U}\left\{\frac{1}{2}\left\|y-y_{d}\right\|_{W}^{2}+\frac{1}{2}\|u\|_{U}^{2}\right\}, \\
& a(\boldsymbol{p}, \boldsymbol{v})-(y, \operatorname{div} \boldsymbol{v})=0, \quad \forall \boldsymbol{v} \in \boldsymbol{V}_{0}, \\
& (\operatorname{div} \boldsymbol{p}, w)+(c y, w)=(f+u, w), \quad \forall w \in W,
\end{aligned}
$$

where $(\cdot, \cdot)$ denotes the stands $L^{2}(\Omega)$ inner product, and $a(\cdot, \cdot)$ is a bilinear form defined by

$$
a(\boldsymbol{p}, \mathbf{v})=\int_{\Omega} A^{-1} \boldsymbol{p} \cdot \mathbf{v} d \Omega .
$$

It is well known (see, e.g., [27]) that the optimal control problem (6)-(8) has a unique one solution $(\boldsymbol{p}, y, u)$, and that a triplet $(\boldsymbol{p}, y, u)$ is the solution of (6)-(8) if and only if there is a co-state $(\boldsymbol{q}, z) \in \boldsymbol{V}_{0} \times W$ such that $(\boldsymbol{p}, y, \boldsymbol{q}, z, u)$ satisfies the following optimality conditions: (CCP-OPT)

$$
\begin{aligned}
& a(\boldsymbol{p}, \boldsymbol{v})-(y, \operatorname{div} \boldsymbol{v})=0, \quad \forall \boldsymbol{v} \in \boldsymbol{V}_{0}, \\
& (\operatorname{div} \boldsymbol{p}, w)+(c y, w)=(f+u, w), \quad \forall w \in W, \\
& a(\boldsymbol{q}, \boldsymbol{v})-(z, \operatorname{div} \boldsymbol{v})=0, \quad \forall \boldsymbol{v} \in \boldsymbol{V}_{0}, \\
& (\operatorname{div} \boldsymbol{q}, w)+(c z, w)=\left(y-y_{d}, w\right), \quad \forall w \in W, \\
& (u+z, \tilde{u}-u)_{U} \geq 0, \quad \forall \tilde{u} \in K .
\end{aligned}
$$

In this paper, we only consider the unconstrained case, that is, $K=U=$ $L^{2}(\Omega)$, which is the special simple case. Thus, it is easy to deduce from (13) that $u=-z$. Then (9)-(13) can be rewritten into

$$
\begin{aligned}
& a(\boldsymbol{p}, \boldsymbol{v})-(y, \operatorname{div} \boldsymbol{v})=0, \quad \forall \boldsymbol{v} \in \boldsymbol{V}_{0}, \\
& (\operatorname{div} \boldsymbol{p}, w)+(c y, w)+(z, w)=(f, w), \quad \forall w \in W, \\
& a(\boldsymbol{q}, \boldsymbol{v})-(z, \operatorname{div} \boldsymbol{v})=0, \quad \forall \boldsymbol{v} \in \boldsymbol{V}_{0}, \\
& (\operatorname{div} \boldsymbol{q}, w)+(c z, w)-(y, w)=\left(-y_{d}, w\right), \quad \forall w \in W,
\end{aligned}
$$

which is the mixed weak form of the following problem:

$$
\begin{aligned}
& -\operatorname{div}(A \nabla y)+c y+z=f, \quad x \in \Omega, \\
& A \nabla y \cdot \boldsymbol{n}=0, \quad x \in \partial \Omega, \\
& -\operatorname{div}(A \nabla z)+c z-y=-y_{d}, \quad x \in \Omega, \\
& A \nabla z \cdot \boldsymbol{n}=0, \quad x \in \partial \Omega .
\end{aligned}
$$

Now, let us consider the mixed finite element approximation to (14)-(17). Let $\mathcal{T}_{h_{1}, h_{2}}$ to be a finite element partition of $\Omega$ into uniform triangles and $\boldsymbol{V}_{h_{1}, h_{2}} \times W_{h_{1}, h_{2}} \subset \boldsymbol{V} \times W$ denote a pair of finite element spaces satisfying the Babuška-Brezzi condition, where $h_{1}$ and $h_{2}$ are the mesh sizes in the $x$ - and $y$ axis, respectively. Even if there are now several choices for $\boldsymbol{V}_{h_{1}, h_{2}}$ and $W_{h_{1}, h_{2}}$, here we will consider only the Raviart-Thomas space of the lowest order; i.e.,

$$
\boldsymbol{V}_{h_{1}, h_{2}}:=\left\{\boldsymbol{v}_{h_{1}, h_{2}} \in \boldsymbol{V}:\left.\boldsymbol{v}_{h_{1}, h_{2}}\right|_{e} \in Q_{1,0}(e) \times Q_{0,1}(e), e \in \mathcal{T}_{h_{1}, h_{2}}\right\},
$$




$$
W_{h_{1}, h_{2}}:=\left\{w_{h_{1}, h_{2}} \in W:\left.w_{h_{1}, h_{2}}\right|_{e} \in Q_{0,0}(e), e \in \mathcal{T}_{h_{1}, h_{2}}\right\},
$$

where $Q_{m, n}(e)$ indicates the space of polynomials of degree no more than $m$ and $n$ in $x$ and $y$ on $e$, respectively. The extension to other stable rectangular element spaces can also be made. Moreover, we let

$$
\boldsymbol{V}_{0, h_{1}, h_{2}}=\boldsymbol{V}_{h_{1}, h_{2}} \cap \boldsymbol{V}_{0} \text {. }
$$

Then the corresponding mixed finite element discretization of (14)-(17) is as follows: compute $\left(y_{h_{1}, h_{2}}, \boldsymbol{p}_{h_{1}, h_{2}}, z_{h_{1}, h_{2}}, \boldsymbol{q}_{h_{1}, h_{2}}\right) \in\left(W_{h_{1}, h_{2}} \times \boldsymbol{V}_{0, h_{1}, h_{2}}\right)^{2} \subset(W \times$ $\left.V_{0}\right)^{2}$ such that

(20) $a\left(\boldsymbol{p}_{h_{1}, h_{2}}, \boldsymbol{v}\right)-\left(y_{h_{1}, h_{2}}, \operatorname{div} \boldsymbol{v}\right)=0, \quad \forall \boldsymbol{v} \in \boldsymbol{V}_{0, h_{1}, h_{2}}$,

(21) $\quad\left(\operatorname{div} \boldsymbol{p}_{h_{1}, h_{2}}, w\right)+\left(c y_{h_{1}, h_{2}}, w\right)+\left(z_{h_{1}, h_{2}}, w\right)=(f, w), \quad \forall w \in W_{h_{1}, h_{2}}$,

(22) $\quad a\left(\boldsymbol{q}_{h_{1}, h_{2}}, \boldsymbol{v}\right)-\left(z_{h_{1}, h_{2}}, \operatorname{div} \boldsymbol{v}\right)=0, \quad \forall \boldsymbol{v} \in \boldsymbol{V}_{0, h_{1}, h_{2}}$,

(23) $\left(\operatorname{div} \boldsymbol{q}_{h_{1}, h_{2}}, w\right)+\left(c z_{h_{1}, h_{2}}, w\right)-\left(y_{h_{1}, h_{2}}, w\right)=\left(-y_{d}, w\right), \quad \forall w \in W_{h_{1}, h_{2}}$.

Let us recall the Raviart-Thomas projection

$$
\Pi_{h_{1}, h_{2}}^{0} \times P_{h_{1}, h_{2}}^{0}: \boldsymbol{V} \times W \rightarrow \boldsymbol{V}_{0, h_{1}, h_{2}} \times W_{h_{1}, h_{2}}
$$

is defined by the following conditions:

$$
\begin{aligned}
& \int_{s_{i}}\left(\mathbf{u}-\Pi_{h_{1}, h_{2}}^{0} \mathbf{u}\right) \cdot \boldsymbol{n} d s=0, \quad i=1,2,3,4, \\
& \int_{e}\left(p-P_{h_{1}, h_{2}}^{0} p\right)=0,
\end{aligned}
$$

where $s_{i}(i=1,2,3,4)$ are the four edges of the rectangle $e \in \mathcal{T}_{h_{1}, h_{2}}$ and $\boldsymbol{n}$ is the outward normal direction on the $s_{i}$. This projection has the following projections [13]:

(i) $P_{h_{1}, h_{2}}^{0}$ is the local $L^{2}(\Omega)$ projection;

(ii) $\Pi_{h_{1}, h_{2}}^{0}$ and $P_{h_{1}, h_{2}}^{0}$ satisfy

$$
\begin{aligned}
& \left(\operatorname{div}\left(\mathbf{u}-\Pi_{h_{1}, h_{2}}^{0} \mathbf{u}\right), w_{h_{1}, h_{2}}\right)=0, \quad \forall w_{h_{1}, h_{2}} \in W_{h_{1}, h_{2}}, \\
& \left(\operatorname{div} \boldsymbol{v}_{h_{1}, h_{2}}, p-P_{h_{1}, h_{2}}^{0} p\right)=0, \quad \forall \boldsymbol{v}_{h_{1}, h_{2}} \in \boldsymbol{V}_{h_{1}, h_{2}} ;
\end{aligned}
$$

(iii) there holds the approximation properties,

$$
\begin{aligned}
& \left\|\mathbf{u}-\Pi_{h_{1}, h_{2}}^{0} \mathbf{u}\right\|_{0} \leq C h\|\mathbf{u}\|_{1}, \\
& \left\|\operatorname{div}\left(\mathbf{u}-\Pi_{h_{1}, h_{2}}^{0} \mathbf{u}\right)\right\|_{-s} \leq C h^{1+s}\|\operatorname{div} \mathbf{u}\|_{1}, \quad 0 \leq s \leq 1, \\
& \left\|p-P_{h_{1}, h_{2}}^{0} p\right\|_{-s} \leq C h^{1+s}\|p\|_{1}, \quad 0 \leq s \leq 1,
\end{aligned}
$$

where $h:=\max \left\{h_{1}, h_{2}\right\}$.

Also, from [15], we recall the following two lemmas to conclude the section.

Lemma 2.1. Assume that $\mathbf{u} \in \boldsymbol{V} \cap\left(H^{4}(\Omega)\right)^{2}$ and $\alpha_{i j} \in H^{4}(\Omega)(1 \leq i, j \leq 2)$. Then we have

$$
\left(\alpha \cdot\left(\mathbf{u}-\Pi_{h_{1}, h_{2}}^{0} \mathbf{u}\right), \boldsymbol{v}\right)=-\frac{h_{1}^{2}}{3} \int_{\Omega}\left[\alpha_{11}\left(u_{1}\right)_{x x}+\alpha_{12}\left(u_{2}\right)_{x x}\right] v_{1} d \Omega
$$




$$
\begin{aligned}
& +\frac{h_{1}^{2}}{3} \int_{\Omega}\left[\left(\alpha_{22}\right)_{x}\left(u_{2}\right)_{x}-\alpha_{21}\left(u_{1}\right)_{x x}\right] v_{2} d \Omega \\
& +\frac{h_{2}^{2}}{3} \int_{\Omega}\left[\left(\alpha_{11}\right)_{y}\left(u_{1}\right)_{y}-\alpha_{12}\left(u_{2}\right)_{y y}\right] v_{1} d \Omega \\
& -\frac{h_{2}^{2}}{3} \int_{\Omega}\left[\alpha_{22}\left(u_{2}\right)_{y y}+\alpha_{21}\left(u_{1}\right)_{y y}\right] v_{2} d \Omega \\
& +O\left(h^{4}\right)\|\mathbf{u}\|_{4}\|\boldsymbol{v}\|_{0}, \quad \boldsymbol{v} \in \boldsymbol{V}_{0, h_{1}, h_{2}}
\end{aligned}
$$

where $u_{1}, u_{2}$ and $v_{1}, v_{2}$ are the first components and the second components of the vector-valued functions $\mathbf{u}$ and $\boldsymbol{v}$, respectively, and $\alpha=\left(\alpha_{i j}\right)_{2 \times 2}$ is the inverse of the matrix $A: \alpha=A^{-1}$.

Lemma 2.2. Assume that $p, c \in H^{3}(\Omega)$. Then we have the asymptotic expansion:

$$
\begin{aligned}
\left(c\left(p-P_{h_{1}, h_{2}}^{0} p\right), w\right)= & \frac{h_{1}^{2}}{3} \int_{\Omega} c_{x} p_{x} w d \Omega+\frac{h_{2}^{2}}{3} \int_{\Omega} c_{y} p_{y} w d \Omega \\
& +O\left(h^{4}\right)\|p\|_{3}\|w\|_{0}, \quad w \in W_{h_{1}, h_{2}} .
\end{aligned}
$$

From Lemmas 2.1 and 2.2 we immediately obtain:

Corollary 2.3. Assume that $\mathbf{u} \in \boldsymbol{V} \cap\left(H^{2}(\Omega)\right)^{2}$ and $\alpha_{i j} \in H^{2}(\Omega)(1 \leq i, j \leq 2)$. Then we have

$$
\left|\left(\alpha \cdot\left(\mathbf{u}-\Pi_{h_{1}, h_{2}}^{0} \mathbf{u}\right), \boldsymbol{v}\right)\right| \leq C h^{2}\|\mathbf{u}\|_{2}\|\boldsymbol{v}\|_{0}, \quad \boldsymbol{v} \in \boldsymbol{V}_{0, h_{1}, h_{2}} .
$$

Corollary 2.4. Assume that $p, c \in H^{1}(\Omega)$. Then we have the asymptotic expansion:

$$
\left|\left(c\left(p-P_{h_{1}, h_{2}}^{0} p\right), w\right)\right| \leq C h^{2}\|p\|_{1}\|w\|_{0}, \quad w \in W_{h_{1}, h_{2}} .
$$

\section{The Richardson extrapolation}

In this section we turn to the asymptotic expansions between the mixed finite element solution and the interpolation of the exact solution of the problem (14)-(17), from which asymptotic expansions between the exact solution and the postprocessed mixed finite element solution by interpolation are further obtained. The Richardson extrapolations of two different schemes will be performed to generate high order approximations to the exact solution of (14)-(17).

\subsection{The global Richardson extrapolation in two directions}

We first discuss the global extrapolation method of mixed finite element approximation for (14)-(17) in both $x$ and $y$ directions as follows.

Theorem 3.1. Suppose that $(y, \boldsymbol{p}, z, \boldsymbol{q})$ and $\left(y_{h_{1}, h_{2}}, \boldsymbol{p}_{h_{1}, h_{2}}, z_{h_{1}, h_{2}}, \boldsymbol{q}_{h_{1}, h_{2}}\right)$ are the exact solution and the mixed finite element solution, respectively. Then we have the following asymptotic expansions in the sense of the $L^{2}$-norm under 
the conditions that $y, z, c \in H^{3}(\Omega), \boldsymbol{p}, \boldsymbol{q} \in \boldsymbol{V} \cap\left(H^{4}(\Omega)\right)^{2}$, and $\alpha_{i j} \in H^{4}(\Omega)(1 \leq$ $i, j \leq 2)$ :

$$
\begin{aligned}
& y_{h_{1}, h_{2}}-P_{h_{1}, h_{2}}^{0} y=h^{2} \xi_{h_{1}, h_{2}}^{1}+\gamma_{h_{1}, h_{2}}^{1}, \quad\left\|\gamma_{h_{1}, h_{2}}^{1}\right\|_{0} \leq C h^{4}, \\
& z_{h_{1}, h_{2}}-P_{h_{1}, h_{2}}^{0} z=h^{2} \xi_{h_{1}, h_{2}}^{2}+\gamma_{h_{1}, h_{2}}^{2}, \quad\left\|\gamma_{h_{1}, h_{2}}^{2}\right\|_{0} \leq C h^{4}, \\
& \boldsymbol{p}_{h_{1}, h_{2}}-\Pi_{h_{1}, h_{2}}^{0} \boldsymbol{p}=h^{2} \eta_{h_{1}, h_{2}}^{1}+\gamma_{h_{1}, h_{2}}^{1}, \quad\left\|\gamma_{h_{1}, h_{2}}^{1}\right\|_{\mathbf{v}} \leq C h^{4}, \\
& \boldsymbol{q}_{h_{1}, h_{2}}-\Pi_{h_{1}, h_{2}}^{0} \boldsymbol{q}=h^{2} \eta_{h_{1}, h_{2}}^{2}+\gamma_{h_{1}, h_{2}}^{2}, \quad\left\|\gamma_{h_{1}, h_{2}}^{2}\right\|_{\mathbf{v}} \leq C h^{4},
\end{aligned}
$$

where $\left(\xi_{h_{1}, h_{2}}^{1}, \eta_{h_{1}, h_{2}}^{1}, \xi_{h_{1}, h_{2}}^{2}, \eta_{h_{1}, h_{2}}^{2}\right) \in\left(W_{h_{1}, h_{2}} \times \boldsymbol{V}_{0, h_{1}, h_{2}}\right)^{2}$ and $\Pi_{h_{1}, h_{2}}^{0} \times P_{h_{1}, h_{2}}^{0}$ : $\boldsymbol{V} \times W \rightarrow \boldsymbol{V}_{0, h_{1}, h_{2}} \times W_{h_{1}, h_{2}}$ is the Raviart-Thomas projection operator.

Proof. Let

$$
\begin{aligned}
& \rho_{h_{1}, h_{2}}^{1}:=y_{h_{1}, h_{2}}-P_{h_{1}, h_{2}}^{0} y, \quad \theta_{h_{1}, h_{2}}^{1}:=\boldsymbol{p}_{h_{1}, h_{2}}-\Pi_{h_{1}, h_{2}}^{0} \boldsymbol{p}, \\
& \rho_{h_{1}, h_{2}}^{2}:=z_{h_{1}, h_{2}}-P_{h_{1}, h_{2}}^{0} z, \quad \theta_{h_{1}, h_{2}}^{2}:=\boldsymbol{q}_{h_{1}, h_{2}}-\Pi_{h_{1}, h_{2}}^{0} \boldsymbol{q} .
\end{aligned}
$$

From (14)-(17) and (20)-(23), we have the following error equations:

$$
\begin{aligned}
& a\left(\boldsymbol{p}-\boldsymbol{p}_{h_{1}, h_{2}}, \boldsymbol{v}\right)-\left(y-y_{h_{1}, h_{2}}, \operatorname{div} \boldsymbol{v}\right)=0, \\
& \left(\operatorname{div}\left(\boldsymbol{p}-\boldsymbol{p}_{h_{1}, h_{2}}\right), w\right)+\left(c\left(y-y_{h_{1}, h_{2}}\right), w\right)+\left(z-z_{h_{1}, h_{2}}, w\right)=0, \\
& a\left(\boldsymbol{q}-\boldsymbol{q}_{h_{1}, h_{2}}, \boldsymbol{v}\right)-\left(z-z_{h_{1}, h_{2}}, \operatorname{div} \boldsymbol{v}\right)=0, \\
& \left(\operatorname{div}\left(\boldsymbol{q}-\boldsymbol{q}_{h_{1}, h_{2}}\right), w\right)+\left(c\left(z-z_{h_{1}, h_{2}}\right), w\right)-\left(y-y_{h_{1}, h_{2}}, w\right)=0
\end{aligned}
$$

for all $\boldsymbol{v} \in \boldsymbol{V}_{0, h_{1}, h_{2}}$ and $w \in W_{h_{1}, h_{2}}$. Then, it follows from (31)-(34) and (26)-(27) that

$$
\begin{aligned}
& a\left(\theta_{h_{1}, h_{2}}^{1}, \boldsymbol{v}\right)-\left(\rho_{h_{1}, h_{2}}^{1}, \operatorname{div} \boldsymbol{v}\right)=\left(\alpha\left(\boldsymbol{p}-\Pi_{h_{1}, h_{2}}^{0} \boldsymbol{p}\right), \boldsymbol{v}\right), \\
& \left(\operatorname{div} \theta_{h_{1}, h_{2}}^{1}, w\right)+\left(c \rho_{h_{1}, h_{2}}^{1}, w\right)+\left(\rho_{h_{1}, h_{2}}^{2}, w\right)=\left(c\left(y-P_{h_{1}, h_{2}}^{0} y\right), w\right), \\
& a\left(\theta_{h_{1}, h_{2}}^{2}, \boldsymbol{v}\right)-\left(\rho_{h_{1}, h_{2}}^{2}, \operatorname{div} \boldsymbol{v}\right)=\left(\alpha\left(\boldsymbol{q}-\Pi_{h_{1}, h_{2}}^{0} \boldsymbol{q}\right), \boldsymbol{v}\right), \\
& \left(\operatorname{div} \theta_{h_{1}, h_{2}}^{2}, w\right)+\left(c \rho_{h_{1}, h_{2}}^{2}, w\right)-\left(\rho_{h_{1}, h_{2}}^{1}, w\right)=\left(c\left(z-P_{h_{1}, h_{2}}^{0} z\right), w\right)
\end{aligned}
$$

for all $\boldsymbol{v} \in \boldsymbol{V}_{0, h_{1}, h_{2}}$ and $w \in W_{h_{1}, h_{2}}$, where $\alpha=A^{-1}$. From Lemmas 2.1 and 2.2 we have

$$
\begin{aligned}
& \left(\alpha\left(\boldsymbol{p}-\Pi_{h_{1}, h_{2}}^{0} \boldsymbol{p}\right), \boldsymbol{v}\right)=h^{2} L_{h_{1}, h_{2}}^{1}(\boldsymbol{v})+O\left(h^{4}\right)\|\boldsymbol{v}\|_{0}, \quad \forall \boldsymbol{v} \in \boldsymbol{V}_{0, h_{1}, h_{2}}, \\
& \left(c\left(y-P_{h_{1}, h_{2}}^{0} y\right), w\right)=h^{2} G_{h_{1}, h_{2}}^{1}(w)+O\left(h^{4}\right)\|w\|_{0}, \quad \forall w \in W_{h_{1}, h_{2}}, \\
& \left(\alpha\left(\boldsymbol{q}-\Pi_{h_{1}, h_{2}}^{0} \boldsymbol{q}\right), \boldsymbol{v}\right)=h^{2} L_{h_{1}, h_{2}}^{2}(\boldsymbol{v})+O\left(h^{4}\right)\|\boldsymbol{v}\|_{0}, \quad \forall \boldsymbol{v} \in \boldsymbol{V}_{0, h_{1}, h_{2}}, \\
& \left(c\left(z-P_{h_{1}, h_{2}}^{0} z\right), w\right)=h^{2} G_{h_{1}, h_{2}}^{2}(w)+O\left(h^{4}\right)\|w\|_{0}, \quad \forall w \in W_{h_{1}, h_{2}},
\end{aligned}
$$

where

$$
\begin{aligned}
L_{h_{1}, h_{2}}^{1}(\psi)= & -\frac{1}{3}\left(\frac{h_{1}}{h}\right)^{2} \int_{\Omega}\left[\alpha_{11}\left(p_{1}\right)_{x x}+\alpha_{12}\left(p_{2}\right)_{x x}\right] \psi_{1} d \Omega \\
& +\frac{1}{3}\left(\frac{h_{1}}{h}\right)^{2} \int_{\Omega}\left[\left(\alpha_{22}\right)_{x}\left(p_{2}\right)_{x}-\alpha_{21}\left(p_{1}\right)_{x x}\right] \psi_{2} d \Omega
\end{aligned}
$$




$$
\begin{aligned}
+ & \frac{1}{3}\left(\frac{h_{2}}{h}\right)^{2} \int_{\Omega}\left[\left(\alpha_{11}\right)_{y}\left(p_{1}\right)_{y}-\alpha_{12}\left(p_{2}\right)_{y y}\right] \psi_{1} d \Omega \\
& -\frac{1}{3}\left(\frac{h_{2}}{h}\right)^{2} \int_{\Omega}\left[\alpha_{22}\left(p_{2}\right)_{y y}+\alpha_{21}\left(p_{1}\right)_{y y}\right] \psi_{2} d \Omega, \\
L_{h_{1}, h_{2}}^{2}(\psi)= & -\frac{1}{3}\left(\frac{h_{1}}{h}\right)^{2} \int_{\Omega}\left[\alpha_{11}\left(q_{1}\right)_{x x}+\alpha_{12}\left(q_{2}\right)_{x x}\right] \psi_{1} d \Omega \\
& +\frac{1}{3}\left(\frac{h_{1}}{h}\right)^{2} \int_{\Omega}\left[\left(\alpha_{22}\right)_{x}\left(q_{2}\right)_{x}-\alpha_{21}\left(q_{1}\right)_{x x}\right] \psi_{2} d \Omega \\
& +\frac{1}{3}\left(\frac{h_{2}}{h}\right)^{2} \int_{\Omega}\left[\left(\alpha_{11}\right)_{y}\left(q_{1}\right)_{y}-\alpha_{12}\left(q_{2}\right)_{y y}\right] \psi_{1} d \Omega \\
& -\frac{1}{3}\left(\frac{h_{2}}{h}\right)^{2} \int_{\Omega}\left[\alpha_{22}\left(q_{2}\right)_{y y}+\alpha_{21}\left(q_{1}\right)_{y y}\right] \psi_{2} d \Omega, \\
G_{h_{1}, h_{2}}^{1}(\phi)= & \frac{1}{3}\left(\frac{h_{1}}{h}\right)^{2} \int_{\Omega} c_{x} y_{x} \phi d \Omega+\frac{1}{3}\left(\frac{h_{2}}{h}\right)^{2} \int_{\Omega} c_{y} y_{y} \phi d \Omega, \\
G_{h_{1}, h_{2}}^{2}(\phi)= & \frac{1}{3}\left(\frac{h_{1}}{h}\right)^{2} \int_{\Omega} c_{x} z_{x} \phi d \Omega+\frac{1}{3}\left(\frac{h_{2}}{h}\right)^{2} \int_{\Omega} c_{y} z_{y} \phi d \Omega .
\end{aligned}
$$

Here $\boldsymbol{\psi}=\left(\psi_{1}, \psi_{2}\right)$ is a vector-valued function. Obviously,

$$
L_{h_{1} / 2, h_{2} / 2}^{i}(\boldsymbol{\psi})=L_{h_{1}, h_{2}}^{i}(\boldsymbol{\psi}) \text { and } G_{h_{1} / 2, h_{2} / 2}^{i}(\phi)=G_{h_{1}, h_{2}}^{i}(\phi), \quad i=1,2 .
$$

Let $\left(\xi^{1}, \eta^{1}, \xi^{2}, \eta^{2}\right) \in\left(W \times \boldsymbol{V}_{0}\right)^{2}$ and $\left(\xi_{h_{1}, h_{2}}^{1}, \eta_{h_{1}, h_{2}}^{1}, \xi_{h_{1}, h_{2}}^{2}, \eta_{h_{1}, h_{2}}^{2}\right) \in\left(W_{h_{1}, h_{2}} \times\right.$ $\left.\boldsymbol{V}_{0, h_{1}, h_{2}}\right)^{2}$ be the exact solution and the mixed finite element solution, respectively, of the following auxiliary problem:

$$
\begin{aligned}
& a\left(\eta^{1}, \boldsymbol{v}\right)-\left(\xi^{1}, \operatorname{div} \boldsymbol{v}\right)=L_{h_{1}, h_{2}}^{1}(\boldsymbol{v}), \quad \forall \boldsymbol{v} \in \boldsymbol{V}_{0}, \\
& \left(\operatorname{div} \eta^{1}, w\right)+\left(c \xi^{1}, w\right)+\left(\xi^{2}, w\right)=G_{h_{1}, h_{2}}^{1}(w), \quad \forall w \in W, \\
& a\left(\eta^{2}, \boldsymbol{v}\right)-\left(\xi^{2}, \operatorname{div} \boldsymbol{v}\right)=L_{h_{1}, h_{2}}^{2}(\boldsymbol{v}), \quad \forall \boldsymbol{v} \in \boldsymbol{V}_{0}, \\
& \left(\operatorname{div} \eta^{2}, w\right)+\left(c \xi^{2}, w\right)-\left(\xi^{1}, w\right)=G_{h_{1}, h_{2}}^{2}(w), \quad \forall w \in W .
\end{aligned}
$$

Then, from (35)-(46) we can get that

$$
\begin{aligned}
& a\left(\theta_{h_{1}, h_{2}}^{1}-h^{2} \eta_{h_{1}, h_{2}}^{1}, \boldsymbol{v}\right)-\left(\rho_{h_{1}, h_{2}}^{1}-h^{2} \xi_{h_{1}, h_{2}}^{1}, \operatorname{div} \boldsymbol{v}\right)=O\left(h^{4}\right)\|\boldsymbol{v}\|_{0}, \\
&\left(\operatorname{div}\left(\theta_{h_{1}, h_{2}}^{1}-h^{2} \eta_{h_{1}, h_{2}}^{1}\right), w\right)+\left(c\left(\rho_{h_{1}, h_{2}}^{1}-h^{2} \xi_{h_{1}, h_{2}}^{1}\right), w\right) \\
&+\left(\rho_{h_{1}, h_{2}}^{2}-h^{2} \xi_{h_{1}, h_{2}}^{2}, w\right)=O\left(h^{4}\right)\|w\|_{0}, \\
& a\left(\theta_{h_{1}, h_{2}}^{2}-h^{2} \eta_{h_{1}, h_{2}}^{2}, \boldsymbol{v}\right)-\left(\rho_{h_{1}, h_{2}}^{2}-h^{2} \xi_{h_{1}, h_{2}}^{2}, \operatorname{div} \boldsymbol{v}\right)=O\left(h^{4}\right)\|\boldsymbol{v}\|_{0}, \\
&\left(\operatorname{div}\left(\theta_{h_{1}, h_{2}}^{2}-h^{2} \eta_{h_{1}, h_{2}}^{2}\right), w\right)+\left(c\left(\rho_{h_{1}, h_{2}}^{2}-h^{2} \xi_{h_{1}, h_{2}}^{2}\right), w\right) \\
&-\left(\rho_{h_{1}, h_{2}}^{1}-h^{2} \xi_{h_{1}, h_{2}}^{1}, w\right)=O\left(h^{4}\right)\|w\|_{0}
\end{aligned}
$$

for all $\boldsymbol{v} \in \boldsymbol{V}_{0, h_{1}, h_{2}}$ and $w \in W_{h_{1}, h_{2}}$. Set

$$
\hat{\theta}_{h_{1}, h_{2}}^{i}:=\theta_{h_{1}, h_{2}}^{i}-h^{2} \eta_{h_{1}, h_{2}}^{i} \text { and } \quad \hat{\rho}_{h_{1}, h_{2}}^{i}:=\rho_{h_{1}, h_{2}}^{i}-h^{2} \xi_{h_{1}, h_{2}}^{i}, \quad i=1,2 .
$$


Thus, we have

(47) $a\left(\hat{\theta}_{h_{1}, h_{2}}^{1}, \boldsymbol{v}\right)-\left(\hat{\rho}_{h_{1}, h_{2}}^{1}, \operatorname{div} \boldsymbol{v}\right)=O\left(h^{4}\right)\|\boldsymbol{v}\|_{0}, \quad \forall \boldsymbol{v} \in \boldsymbol{V}_{0, h_{1}, h_{2}}$,

(48) $\left(\operatorname{div} \hat{\theta}_{h_{1}, h_{2}}^{1}, w\right)+\left(c \hat{\rho}_{h_{1}, h_{2}}^{1}, w\right)+\left(\hat{\rho}_{h_{1}, h_{2}}^{2}, w\right)=O\left(h^{4}\right)\|w\|_{0}, \quad \forall w \in W_{h_{1}, h_{2}}$,

(49) $a\left(\hat{\theta}_{h_{1}, h_{2}}^{2}, \boldsymbol{v}\right)-\left(\hat{\rho}_{h_{1}, h_{2}}^{2}, \operatorname{div} \boldsymbol{v}\right)=O\left(h^{4}\right)\|\boldsymbol{v}\|_{0}, \quad \forall \boldsymbol{v} \in \boldsymbol{V}_{0, h_{1}, h_{2}}$,

(50) $\left(\operatorname{div} \hat{\theta}_{h_{1}, h_{2}}^{2}, w\right)+\left(c \hat{\rho}_{h_{1}, h_{2}}^{2}, w\right)-\left(\hat{\rho}_{h_{1}, h_{2}}^{1}, w\right)=O\left(h^{4}\right)\|w\|_{0}, \quad \forall w \in W_{h_{1}, h_{2}}$.

Take $\boldsymbol{v}=\hat{\theta}_{h_{1}, h_{2}}^{1}$ in (47), $w=\hat{\rho}_{h_{1}, h_{2}}^{1}$ in (48), $\boldsymbol{v}=\hat{\theta}_{h_{1}, h_{2}}^{2}$ in (49), and $w=\hat{\rho}_{h_{1}, h_{2}}^{2}$ in (50), respectively, we easily obtain

$$
\begin{aligned}
& a\left(\hat{\theta}_{h_{1}, h_{2}}^{1}, \hat{\theta}_{h_{1}, h_{2}}^{1}\right)+a\left(\hat{\theta}_{h_{1}, h_{2}}^{2}, \hat{\theta}_{h_{1}, h_{2}}^{2}\right)+\left(c \hat{\rho}_{h_{1}, h_{2}}^{1}, \hat{\rho}_{h_{1}, h_{2}}^{1}\right)+\left(c \hat{\rho}_{h_{1}, h_{2}}^{2}, \hat{\rho}_{h_{1}, h_{2}}^{2}\right) \\
= & O\left(h^{4}\right)\left(\left\|\hat{\theta}_{h_{1}, h_{2}}^{1}\right\|_{0}+\left\|\hat{\theta}_{h_{1}, h_{2}}^{2}\right\|_{0}+\left\|\hat{\rho}_{h_{1}, h_{2}}^{1}\right\|_{0}+\left\|\hat{\rho}_{h_{1}, h_{2}}^{2}\right\|_{0}\right),
\end{aligned}
$$

yields to

$$
\left\|\hat{\theta}_{h_{1}, h_{2}}^{1}\right\|_{0}+\left\|\hat{\theta}_{h_{1}, h_{2}}^{2}\right\|_{0}+\left\|\hat{\rho}_{h_{1}, h_{2}}^{1}\right\|_{0}+\left\|\hat{\rho}_{h_{1}, h_{2}}^{2}\right\|_{0} \leq C h^{4} .
$$

If we choose $w=\operatorname{div} \hat{\theta}_{h_{1}, h_{2}}^{1}$ in (48), by use of (51), we get

$$
\left\|\operatorname{div} \hat{\theta}_{h_{1}, h_{2}}^{1}\right\|_{0} \leq C h^{4} \text {. }
$$

Similarly, we see that

$$
\left\|\operatorname{div} \hat{\theta}_{h_{1}, h_{2}}^{2}\right\|_{0} \leq C h^{4} .
$$

Thus, the proof of Theorem 3.1 is complete.

Following the procedure for Theorem 3.1 and utilizing Corollaries 2.3 and 2.4 we can also prove the following result.

Lemma 3.2. If $\left(\xi^{1}, \eta^{1}, \xi^{2}, \eta^{2}\right) \in\left(W \times V_{0}\right)^{2}$ and $\left(\xi_{h_{1}, h_{2}}^{1}, \eta_{h_{1}, h_{2}}^{1}, \xi_{h_{1}, h_{2}}^{2}, \eta_{h_{1}, h_{2}}^{2}\right) \in$ $\left(W_{h_{1}, h_{2}} \times \boldsymbol{V}_{0, h_{1}, h_{2}}\right)^{2}$ be the variational solution and the mixed finite element solution of (43)-(46), respectively, then we have the superconvergent estimate

$$
\begin{gathered}
\left\|\xi_{h_{1}, h_{2}}^{1}-P_{h_{1}, h_{2}}^{0} \xi^{1}\right\|_{0}+\left\|\xi_{h_{1}, h_{2}}^{2}-P_{h_{1}, h_{2}}^{0} \xi^{2}\right\|_{0}+\left\|\eta_{h_{1}, h_{2}}^{1}-\Pi_{h_{1}, h_{2}}^{0} \eta^{1}\right\|_{0} \\
+\left\|\eta_{h_{1}, h_{2}}^{2}-\Pi_{h_{1}, h_{2}}^{0} \eta^{2}\right\|_{0} \leq C h^{2}\left(\left\|\eta^{1}\right\|_{2}+\left\|\eta^{2}\right\|_{2}+\left\|\xi^{1}\right\|_{1}+\left\|\xi^{2}\right\|_{1}\right) .
\end{gathered}
$$

Now we use the interpolation postprocessing technique to get a global extrapolation approximation of high accuracy in both $x$ and $y$ directions. Analogous to [22] we need two define two postprocessing interpolation operators $\Pi_{4 h_{1}, 4 h_{2}}^{3}$ and $P_{4 h_{1}, 4 h_{2}}^{3}$ to satisfy

$$
\begin{aligned}
& \Pi_{4 h_{1}, 4 h_{2}}^{3} \Pi_{h_{1}, h_{2}}^{0}=\Pi_{4 h_{1}, 4 h_{2}}^{3}, \\
& \left\|\Pi_{4 h_{1}, 4 h_{2}}^{3} \boldsymbol{v}\right\|_{0} \leq C\|\boldsymbol{v}\|_{0}, \quad \forall \boldsymbol{v} \in \boldsymbol{V}_{0, h_{1}, h_{2}}, \\
& \left\|\Pi_{4 h_{1}, 4 h_{2}}^{3} \mathbf{u}-\mathbf{u}\right\|_{0} \leq C h^{4}\|\mathbf{u}\|_{4}, \quad \forall \mathbf{u} \in\left(H^{4}(\Omega)\right)^{2}, \\
& P_{4 h_{1}, 4 h_{2}}^{3} P_{h_{1}, h_{2}}^{0}=P_{4 h_{1}, 4 h_{2}}^{3}, \\
& \left\|P_{4 h_{1}, 4 h_{2}}^{3} w\right\|_{0} \leq C\|w\|_{0}, \quad \forall w \in W_{h_{1}, h_{2}}, \\
& \left\|P_{4 h_{1}, 4 h_{2}}^{3} p-p\right\|_{0} \leq C h^{4}\|p\|_{4}, \quad \forall p \in H^{4}(\Omega) .
\end{aligned}
$$


To this end, assume that the rectangular partition $\mathcal{T}_{h_{1}, h_{2}}$ has been obtained from $\mathcal{T}_{4 h_{1}, 4 h_{2}}$ with mesh size $4 h$ by subdividing each element of $\mathcal{T}_{4 h_{1}, 4 h_{2}}$ into sixteen small congruent rectangles. Let $\tau:=\bigcup_{i=1}^{16} e_{i}$ with $e_{i} \in \mathcal{T}$. We define two projection operators $\Pi_{4 h_{1}, 4 h_{2}}^{3}$ and $P_{4 h_{1}, 4 h_{2}}^{3}$ associated with $\mathcal{T}_{4 h_{1}, 4 h_{2}}$ of degree at most 3 in $x$ and $y$ on $\tau$, respectively, according to the following conditions:

$$
\begin{aligned}
& \left.\Pi_{4 h_{1}, 4 h_{2}}^{3} \mathbf{u}\right|_{\tau} \in Q_{4,3}(\tau) \times Q_{3,4}(\tau),\left.\quad P_{4 h_{1}, 4 h_{2}}^{3} p\right|_{\tau} \in Q_{3,3}(\tau), \\
& \int_{s_{i}}\left(\mathbf{u}-\Pi_{4 h_{1}, 4 h_{2}}^{3} \mathbf{u}\right) \cdot \boldsymbol{n} d s=0, \quad i=1,2, \ldots, 40, \\
& \int_{e_{i}}\left(p-P_{4 h_{1}, 4 h_{2}}^{3} p\right)=0, \quad i=1,2, \ldots, 16,
\end{aligned}
$$

where $s_{i}(i=1,2, \ldots, 40)$ is one of the forty sides of the sixteen small elements $e_{i}(i=1,2, \ldots, 16)$. It is easy to check that the two operators $\Pi_{4 h_{1}, 4 h_{2}}^{3}$ and $P_{4 h_{1}, 4 h_{2}}^{3}$ defined by (59)-(61) satisfy the properties described in (53)-(58).

Theorem 3.3. We have under the conditions of Theorem 3.1 that

$$
\begin{aligned}
& P_{4 h_{1}, 4 h_{2}}^{3} y_{h_{1}, h_{2}}-y=h^{2} \xi^{1}+\gamma_{h_{1}, h_{2}}^{1 *}, \quad\left\|\gamma_{h_{1}, h_{2}}^{1 *}\right\|_{0} \leq C h^{4}, \\
& P_{4 h_{1}, 4 h_{2}}^{3} z_{h_{1}, h_{2}}-z=h^{2} \xi^{2}+\gamma_{h_{1}, h_{2}}^{2 *}, \quad\left\|\gamma_{h_{1}, h_{2}}^{2 *}\right\|_{0} \leq C h^{4}, \\
& \Pi_{4 h_{1}, 4 h_{2}}^{3} \boldsymbol{p}_{h_{1}, h_{2}}-\boldsymbol{p}=h^{2} \eta^{1}+\gamma_{h_{1}, h_{2}}^{1 *}, \quad\left\|\gamma_{h_{1}, h_{2}}^{1 *}\right\|_{0} \leq C h^{4}, \\
& \Pi_{4 h_{1}, 4 h_{2}}^{3} \boldsymbol{q}_{h_{1}, h_{2}}-\boldsymbol{q}=h^{2} \eta^{2}+\gamma_{h_{1}, h_{2}}^{2 *}, \quad\left\|\gamma_{h_{1}, h_{2}}^{2 *}\right\|_{0} \leq C h^{4},
\end{aligned}
$$

where $\left(\xi^{1}, \eta^{1}, \xi^{2}, \eta^{2}\right) \in\left(W \times \boldsymbol{V}_{0}\right)^{2}$ is the variational solution of (43)-(46).

Proof. Let

$$
\bar{\gamma}_{h_{1}, h_{2}}^{1}:=y_{h_{1}, h_{2}}-P_{h_{1}, h_{2}}^{0} y-h^{2} P_{h_{1}, h_{2}}^{0} \xi^{1} .
$$

Then, it follows from Theorem 3.1 and Lemma 3.2 that

$$
\left\|\bar{\gamma}_{h_{1}, h_{2}}^{1}\right\|_{0} \leq C h^{4} \text {. }
$$

Thus, we will find from (56)-(58) that

$$
\begin{aligned}
& P_{4 h_{1}, 4 h_{2}}^{3} y_{h_{1}, h_{2}}-y \\
= & P_{4 h_{1}, 4 h_{2}}^{3}\left(y_{h_{1}, h_{2}}-P_{h_{1}, h_{2}}^{0} y\right)+\left(P_{4 h_{1}, 4 h_{2}}^{3} y-y\right) \\
= & P_{4 h_{1}, 4 h_{2}}^{3}\left(h^{2} P_{h_{1}, h_{2}}^{0} \xi^{1}+\bar{\gamma}_{h_{1}, h_{2}}^{1}\right)+\left(P_{4 h_{1}, 4 h_{2}}^{3} y-y\right) \\
= & h^{2} P_{4 h_{1}, 4 h_{2}}^{3} \xi^{1}+P_{4 h_{1}, 4 h_{2}}^{3} \bar{\gamma}_{h_{1}, h_{2}}^{1}+\left(P_{4 h_{1}, 4 h_{2}}^{3} y-y\right) \\
= & h^{2} \xi^{1}+h^{2}\left(P_{4 h_{1}, 4 h_{2}}^{3} \xi^{1}-\xi^{1}\right)+P_{4 h_{1}, 4 h_{2}}^{3} \bar{\gamma}_{h_{1}, h_{2}}^{1}+\left(P_{4 h_{1}, 4 h_{2}}^{3} y-y\right) \\
= & h^{2} \xi^{1}+\gamma_{h_{1}, h_{2}}^{1 *},
\end{aligned}
$$

where

$$
\gamma_{h_{1}, h_{2}}^{1 *}:=h^{2}\left(P_{4 h_{1}, 4 h_{2}}^{3} \xi^{1}-\xi^{1}\right)+P_{4 h_{1}, 4 h_{2}}^{3} \bar{\gamma}_{h_{1}, h_{2}}^{1}+\left(P_{4 h_{1}, 4 h_{2}}^{3} y-y\right)
$$

with $\left\|\gamma_{h_{1}, h_{2}}^{1 *}\right\|_{0} \leq C h^{4}$. Analogously, we can also get other equalities in the theorem. 
Theorem 3.3 guarantees that we can use low order mixed finite element solutions to generate high order approximations by the Richardson extrapolation. And thus, we employ, in addition to $W_{h_{1}, h_{2}} \times \boldsymbol{V}_{0, h_{1}, h_{2}}$ the Raviart-Thomas mixed finite element space $W_{h_{1} / 2, h_{2} / 2} \times \boldsymbol{V}_{0, h_{1} / 2, h_{2} / 2}$ of the lowest order gained by subdividing each element $e_{i} \in \mathcal{T}_{h_{1}, h_{2}}$ into four small congruent elements $\hat{e}_{i, j} \in \mathcal{T}_{h_{1} / 2, h_{2} / 2}(j=1,2,3,4)$. Denote by $\left(y_{h_{1} / 2, h_{2} / 2}, \boldsymbol{p}_{h_{1} / 2, h_{2} / 2}, z_{h_{1} / 2, h_{2} / 2}\right.$, $\left.\boldsymbol{q}_{h_{1} / 2, h_{2} / 2}\right) \in\left(W_{h_{1} / 2, h_{2} / 2} \times \boldsymbol{V}_{0, h_{1} / 2, h_{2} / 2}\right)^{2}$ and $\Pi_{2 h_{1}, 2 h_{2}}^{3} \times P_{2 h_{1}, 2 h_{2}}^{3}$ the mixed finite element approximation and the Raviart-Thomas projection of degree at most 3 in $x$ and $y$ with respect to this new partition. From Theorem 3.3 we know under the $L^{2}$-norm that

$$
P_{2 h_{1}, 2 h_{2}}^{3} y_{h_{1} / 2, h_{2} / 2}-y=\left(\frac{h}{2}\right)^{2} \xi^{1}+O\left(h^{4}\right),
$$

which produces by applying the Richardson extrapolation that under the $L^{2}$ norm

$$
\frac{4 P_{2 h_{1}, 2 h_{2}}^{3} y_{h_{1} / 2, h_{2} / 2}-P_{4 h_{1}, 4 h_{2}}^{3} y_{h_{1}, h_{2}}}{3}=y+O\left(h^{4}\right) .
$$

Similarly, we have under the $L^{2}$-norm that

$$
\begin{aligned}
& \frac{4 P_{2 h_{1}, 2 h_{2}}^{3} z_{h_{1} / 2, h_{2} / 2}-P_{4 h_{1}, 4 h_{2}}^{3} z_{h_{1}, h_{2}}}{3}=z+O\left(h^{4}\right), \\
& \frac{4 \Pi_{2 h_{1}, 2 h_{2}}^{3} \boldsymbol{p}_{h_{1} / 2, h_{2} / 2}-P_{4 h_{1}, 4 h_{2}}^{3} \boldsymbol{p}_{h_{1}, h_{2}}}{3}=\boldsymbol{p}+O\left(h^{4}\right), \\
& \frac{4 \Pi_{2 h_{1}, 2 h_{2}}^{3} \boldsymbol{q}_{h_{1} / 2, h_{2} / 2}-P_{4 h_{1}, 4 h_{2}}^{3} \boldsymbol{q}_{h_{1}, h_{2}}}{3}=\boldsymbol{q}+O\left(h^{4}\right) .
\end{aligned}
$$

It is very important for a mixed finite element method to have a computable a posteriori error estimator such that we can assess the accuracy of the approximate solutions by means of the error estimator in applications. The superconvergent approximations generated above in (62)-(65) can be used naturally to produce efficient a posteriori error estimators. In fact, we have by the same way as in Theorem 5.3 in [14] that the following theorem holds.

Theorem 3.4. Under the assumptions of Theorem 3.3, we have

$$
\begin{aligned}
& \left\|y-P_{2 h_{1}, 2 h_{2}}^{3} y_{h_{1} / 2, h_{2} / 2}\right\|_{0} \\
= & \frac{1}{3}\left\|P_{2 h_{1}, 2 h_{2}}^{3} y_{h_{1} / 2, h_{2} / 2}-P_{4 h_{1}, 4 h_{2}}^{3} y_{h_{1}, h_{2}}\right\|_{0}+O\left(h^{4}\right), \\
& \left\|z-P_{2 h_{1}, 2 h_{2}}^{3} z_{h_{1} / 2, h_{2} / 2}\right\|_{0} \\
= & \frac{1}{3}\left\|P_{2 h_{1}, 2 h_{2}}^{3} z_{h_{1} / 2, h_{2} / 2}-P_{4 h_{1}, 4 h_{2}}^{3} z_{h_{1}, h_{2}}\right\|_{0}+O\left(h^{4}\right), \\
& \left\|\boldsymbol{p}-\Pi_{2 h_{1}, 2 h_{2}}^{3} \boldsymbol{p}_{h_{1} / 2, h_{2} / 2}\right\|_{0} \\
= & \frac{1}{3}\left\|\Pi_{2 h_{1}, 2 h_{2}}^{3} \boldsymbol{p}_{h_{1} / 2, h_{2} / 2}-\Pi_{4 h_{1}, 4 h_{2}}^{3} \boldsymbol{p}_{h_{1}, h_{2}}\right\|_{0}+O\left(h^{4}\right),
\end{aligned}
$$




$$
\begin{aligned}
& \left\|\boldsymbol{q}-\Pi_{2 h_{1}, 2 h_{2}}^{3} \boldsymbol{q}_{h_{1} / 2, h_{2} / 2}\right\|_{0} \\
= & \frac{1}{3}\left\|\Pi_{2 h_{1}, 2 h_{2}}^{3} \boldsymbol{q}_{h_{1} / 2, h_{2} / 2}-\Pi_{4 h_{1}, 4 h_{2}}^{3} \boldsymbol{q}_{h_{1}, h_{2}}\right\|_{0}+O\left(h^{4}\right) .
\end{aligned}
$$

In addition, if there exist positive constants $C_{1}, C_{2}, C_{3}, C_{4}$ and $\epsilon_{1}, \epsilon_{2}, \epsilon_{3}, \epsilon_{4} \in$ $(0,1)$ such that

then we have

$$
\begin{aligned}
& \left\|y-P_{2 h_{1}, 2 h_{2}}^{3} y_{h_{1} / 2, h_{2} / 2}\right\|_{0} \geq C_{1} h^{4-\epsilon_{1}}, \\
& \left\|z-P_{2 h_{1}, 2 h_{2}}^{3} z_{h_{1} / 2, h_{2} / 2}\right\|_{0} \geq C_{2} h^{4-\epsilon_{2}}, \\
& \left\|\boldsymbol{p}-\Pi_{2 h_{1}, 2 h_{2}}^{3} \boldsymbol{p}_{h_{1} / 2, h_{2} / 2}\right\|_{0} \geq C_{3} h^{4-\epsilon_{3}}, \\
& \left\|\boldsymbol{q}-P_{2 h_{1}, 2 h_{2}}^{3} \boldsymbol{q}_{h_{1} / 2, h_{2} / 2}\right\|_{0} \geq C_{4} h^{4-\epsilon_{4},},
\end{aligned}
$$

$$
\begin{aligned}
& \lim _{h \rightarrow 0} \frac{3\left\|y-P_{2 h_{1}, 2 h_{2}}^{3} y_{h_{1} / 2, h_{2} / 2}\right\|_{0}}{\left\|P_{2 h_{1}, 2 h_{2}}^{3} y_{h_{1} / 2, h_{2} / 2}-P_{4 h_{1}, 4 h_{2}}^{3} y_{h_{1}, h_{2}}\right\|_{0}}=1, \\
& \lim _{h \rightarrow 0} \frac{3\left\|z-P_{2 h_{1}, 2 h_{2}}^{3} z_{h_{1} / 2, h_{2} / 2}\right\|_{0}}{\left\|P_{2 h_{1}, 2 h_{2}}^{3} z_{h_{1} / 2, h_{2} / 2}-P_{4 h_{1}, 4 h_{2}}^{3} z_{h_{1}, h_{2}}\right\|_{0}}=1, \\
& \lim _{h \rightarrow 0} \frac{3\left\|\boldsymbol{p}-\Pi_{2 h_{1}, 2 h_{2}}^{3} \boldsymbol{p}_{h_{1} / 2, h_{2} / 2}\right\|_{0}}{\left\|\Pi_{2 h_{1}, 2 h_{2}}^{3} \boldsymbol{p}_{h_{1} / 2, h_{2} / 2}-\Pi_{4 h_{1}, 4 h_{2}}^{3} \boldsymbol{p}_{h_{1}, h_{2}}\right\|_{0}}=1, \\
& \lim _{h \rightarrow 0} \frac{3\left\|\boldsymbol{q}-\Pi_{2 h_{1}, 2 h_{2}}^{3} \boldsymbol{q}_{h_{1} / 2, h_{2} / 2}\right\|_{0}}{\left\|\Pi_{2 h_{1}, 2 h_{2}}^{3} \boldsymbol{q}_{h_{1} / 2, h_{2} / 2}-\Pi_{4 h_{1}, 4 h_{2}}^{3} \boldsymbol{q}_{h_{1}, h_{2}}\right\|_{0}}=1 .
\end{aligned}
$$

From (66) we see that the computable error estimator $\frac{1}{3} \| P_{2 h_{1}, 2 h_{2}}^{3} y_{h_{1} / 2, h_{2} / 2}-$ $P_{4 h_{1}, 4 h_{2}}^{3} y_{h_{1}, h_{2}} \|_{0}$ is the principal part of the error $\left\|y-P_{2 h_{1}, 2 h_{2}}^{3} y_{h_{1} / 2, h_{2} / 2}\right\|_{0}$, and can be used as a posteriori error indicator to assess the accuracy of the pressure error $\left\|y-P_{2 h_{1}, 2 h_{2}}^{3} y_{h_{1} / 2, h_{2} / 2}\right\|_{0}$. Meanwhile, the condition (70) seems to be a reasonable assumption because $O\left(h^{2}\right)$ is the optimal convergence rate of $\| y-$ $P_{2 h_{1}, 2 h_{2}}^{3} y_{h_{1} / 2, h_{2} / 2} \|_{0}$ according to Theorem 3.3. Also, it can be further seen from (74) that the a posteriori error estimator $\frac{1}{3}\left\|P_{2 h_{1}, 2 h_{2}}^{3} y_{h_{1} / 2, h_{2} / 2}-P_{4 h_{1}, 4 h_{2}}^{3} y_{h_{1}, h_{2}}\right\|_{0}$ is quite reliable. The same comments are also valid for (67)-(69), (71)-(73), and (75)-(77).

\subsection{The global Richardson extrapolation in one direction}

The approach introduced in the last subsection has a limitation in that it requires a global and uniform refinement in both the $x$ - and $y$-directions, and hence, it wastes computing time and memory. To overcome this shortcoming, here we propose an extrapolation method of a partial refinement [22], in which the meshes are fined just in either the $x$ - and $y$ - direction. Thus, this method is more efficient and is also more suitable for parallel computations.

Theorem 3.5. Under the conditions of Theorem 3.1, we have in the sense of the $L^{2}$-norm that

$$
y_{h_{1}, h_{2}}-P_{h_{1}, h_{2}}^{0} y=h_{1}^{2} \xi_{h_{1}, h_{2}}^{11}+h_{2}^{2} \xi_{h_{1}, h_{2}}^{12}+r_{h_{1}, h_{2}}^{1}, \quad\left\|r_{h_{1}, h_{2}}^{1}\right\|_{0} \leq C h^{4},
$$




$$
\begin{array}{ll}
z_{h_{1}, h_{2}}-P_{h_{1}, h_{2}}^{0} z=h_{1}^{2} \xi_{h_{1}, h_{2}}^{21}+h_{2}^{2} \xi_{h_{1}, h_{2}}^{22}+r_{h_{1}, h_{2}}^{2}, & \left\|r_{h_{1}, h_{2}}^{2}\right\|_{0} \leq C h^{4}, \\
\boldsymbol{p}_{h_{1}, h_{2}}-\Pi_{h_{1}, h_{2}}^{0} \boldsymbol{p}=h_{1}^{2} \eta_{h_{1}, h_{2}}^{11}+h_{2}^{2} \eta_{h_{1}, h_{2}}^{12}+\boldsymbol{r}_{h_{1}, h_{2}}^{1}, & \left\|\boldsymbol{r}_{h_{1}, h_{2}}^{1}\right\|_{\mathbf{v}} \leq C h^{4}, \\
\boldsymbol{q}_{h_{1}, h_{2}}-\Pi_{h_{1}, h_{2}}^{0} \boldsymbol{q}=h_{1}^{2} \eta_{h_{1}, h_{2}}^{21}+h_{2}^{2} \eta_{h_{1}, h_{2}}^{22}+\boldsymbol{r}_{h_{1}, h_{2}}^{2}, & \left\|\boldsymbol{r}_{h_{1}, h_{2}}^{2}\right\|_{\mathbf{v}} \leq C h^{4},
\end{array}
$$

where $\left(\xi_{h_{1}, h_{2}}^{11}, \eta_{h_{1}, h_{2}}^{11}, \xi_{h_{1}, h_{2}}^{21}, \eta_{h_{1}, h_{2}}^{21}\right),\left(\xi_{h_{1}, h_{2}}^{12}, \eta_{h_{1}, h_{2}}^{12}, \xi_{h_{1}, h_{2}}^{22}, \eta_{h_{1}, h_{2}}^{22}\right) \in\left(W_{h_{1}, h_{2}} \times\right.$ $\left.\boldsymbol{V}_{0, h_{1}, h_{2}}\right)^{2}$ and $\Pi_{h_{1}, h_{2}}^{0} \times P_{h_{1}, h_{2}}^{0}: \boldsymbol{V} \times W \rightarrow \boldsymbol{V}_{0, h_{1}, h_{2}} \times W_{h_{1}, h_{2}}$ is the RaviartThomas projection operator.

Proof. Let $\left(\xi^{11}, \eta^{11}, \xi^{21}, \eta^{21}\right),\left(\xi^{12}, \eta^{12}, \xi^{22}, \eta^{22}\right) \in\left(W \times \boldsymbol{V}_{0}\right)^{2}$ and $\left(\xi_{h_{1}, h_{2}}^{11}, \eta_{h_{1}, h_{2}}^{11}\right.$, $\left.\xi_{h_{1}, h_{2}}^{21}, \eta_{h_{1}, h_{2}}^{21}\right),\left(\xi_{h_{1}, h_{2}}^{12}, \eta_{h_{1}, h_{2}}^{12}, \xi_{h_{1}, h_{2}}^{22}, \eta_{h_{1}, h_{2}}^{22}\right) \in\left(W_{h_{1}, h_{2}} \times V_{0, h_{1}, h_{2}}\right)^{2}$ be the exact solutions and the mixed finite element solutions, respectively, of the following two auxiliary variational problem:

$$
\begin{aligned}
& a\left(\eta^{11}, \boldsymbol{v}\right)-\left(\xi^{11}, \operatorname{div} \boldsymbol{v}\right)=L_{1}(\boldsymbol{v}), \quad \forall \boldsymbol{v} \in \boldsymbol{V}_{0}, \\
& \left(\operatorname{div} \eta^{11}, w\right)+\left(c \xi^{11}, w\right)+\left(\xi^{21}, w\right)=L_{5}(w), \quad \forall w \in W, \\
& a\left(\eta^{21}, \boldsymbol{v}\right)-\left(\xi^{21}, \operatorname{div} \boldsymbol{v}\right)=L_{2}(\boldsymbol{v}), \quad \forall \boldsymbol{v} \in \boldsymbol{V}_{0}, \\
& \left(\operatorname{div} \eta^{21}, w\right)+\left(c \xi^{21}, w\right)-\left(\xi^{11}, w\right)=L_{6}(w), \quad \forall w \in W,
\end{aligned}
$$

and

$$
\begin{aligned}
& a\left(\eta^{12}, \boldsymbol{v}\right)-\left(\xi^{12}, \operatorname{div} \boldsymbol{v}\right)=L_{3}(\boldsymbol{v}), \quad \forall \boldsymbol{v} \in \boldsymbol{V}_{0}, \\
& \left(\operatorname{div} \eta^{12}, w\right)+\left(c \xi^{12}, w\right)+\left(\xi^{22}, w\right)=L_{7}(w), \quad \forall w \in W, \\
& a\left(\eta^{22}, \boldsymbol{v}\right)-\left(\xi^{22}, \operatorname{div} \boldsymbol{v}\right)=L_{4}(\boldsymbol{v}), \quad \forall \boldsymbol{v} \in \boldsymbol{V}_{0}, \\
& \left(\operatorname{div} \eta^{22}, w\right)+\left(c \xi^{22}, w\right)-\left(\xi^{12}, w\right)=L_{8}(w), \quad \forall w \in W,
\end{aligned}
$$

where

$$
\begin{aligned}
L_{1}(\boldsymbol{v})= & -\frac{1}{3} \int_{\Omega}\left[\alpha_{11}\left(p_{1}\right)_{x x}+\alpha_{12}\left(p_{2}\right)_{x x}\right] v_{1} d \Omega \\
& +\frac{1}{3} \int_{\Omega}\left[\left(\alpha_{22}\right)_{x}\left(p_{2}\right)_{x}-\alpha_{21}\left(p_{1}\right)_{x x}\right] v_{2} d \Omega, \\
L_{2}(\boldsymbol{v})= & -\frac{1}{3} \int_{\Omega}\left[\alpha_{11}\left(q_{1}\right)_{x x}+\alpha_{12}\left(q_{2}\right)_{x x}\right] v_{1} d \Omega \\
& +\frac{1}{3} \int_{\Omega}\left[\left(\alpha_{22}\right)_{x}\left(q_{2}\right)_{x}-\alpha_{21}\left(q_{1}\right)_{x x}\right] v_{2} d \Omega, \\
L_{3}(\boldsymbol{v})= & \frac{1}{3} \int_{\Omega}\left[\left(\alpha_{11}\right)_{y}\left(p_{1}\right)_{y}-\alpha_{12}\left(p_{2}\right)_{y y}\right] v_{1} d \Omega \\
& -\frac{1}{3} \int_{\Omega}\left[\alpha_{22}\left(p_{2}\right)_{y y}+\alpha_{21}\left(p_{1}\right)_{y y}\right] v_{2} d \Omega, \\
L_{4}(\boldsymbol{v})= & \frac{1}{3} \int_{\Omega}\left[\left(\alpha_{11}\right)_{y}\left(q_{1}\right)_{y}-\alpha_{12}\left(q_{2}\right)_{y y}\right] v_{1} d \Omega \\
& -\frac{1}{3} \int_{\Omega}\left[\alpha_{22}\left(q_{2}\right)_{y y}+\alpha_{21}\left(q_{1}\right)_{y y}\right] v_{2} d \Omega,
\end{aligned}
$$




$$
\begin{aligned}
L_{5}(w) & =\frac{1}{3} \int_{\Omega} c_{x} y_{x} \phi d \Omega, & L_{6}(w) & =\frac{1}{3} \int_{\Omega} c_{x} z_{x} \phi d \Omega, \\
L_{7}(w) & =\frac{1}{3} \int_{\Omega} c_{y} y_{y} \phi d \Omega, & L_{8}(w) & =\frac{1}{3} \int_{\Omega} c_{y} z_{y} \phi d \Omega .
\end{aligned}
$$

Then, it follows from (35)-(38) and Lemmas 2.1 and 2.2 that

$$
\begin{aligned}
& a\left(\theta_{h_{1}, h_{2}}^{1}, \boldsymbol{v}\right)-\left(\rho_{h_{1}, h_{2}}^{1}, \operatorname{div} \boldsymbol{v}\right)=h_{1}^{2} L_{1}(\boldsymbol{v})+h_{2}^{2} L_{3}(\boldsymbol{v})+O\left(h^{4}\right)\|\boldsymbol{v}\|_{0}, \\
& \left(\operatorname{div} \theta_{h_{1}, h_{2}}^{1}, w\right)+\left(c \rho_{h_{1}, h_{2}}^{1}, w\right)+\left(\rho_{h_{1}, h_{2}}^{2}, w\right) \\
= & h_{1}^{2} L_{5}(w)+h_{2}^{2} L_{7}(w)+O\left(h^{4}\right)\|w\|_{0}, \\
& a\left(\theta_{h_{1}, h_{2}}^{2}, \boldsymbol{v}\right)-\left(\rho_{h_{1}, h_{2}}^{2}, \operatorname{div} \boldsymbol{v}\right)=h_{1}^{2} L_{2}(\boldsymbol{v})+h_{2}^{2} L_{4}(\boldsymbol{v})+O\left(h^{4}\right)\|\boldsymbol{v}\|_{0}, \\
& \left(\operatorname{div} \theta_{h_{1}, h_{2}}^{2}, w\right)+\left(c \rho_{h_{1}, h_{2}}^{2}, w\right)-\left(\rho_{h_{1}, h_{2}}^{1}, w\right) \\
= & h_{1}^{2} L_{6}(w)+h_{2}^{2} L_{8}(w)+O\left(h^{4}\right)\|w\|_{0}
\end{aligned}
$$

for all $\boldsymbol{v} \in \boldsymbol{V}_{0, h_{1}, h_{2}}$ and $w \in W_{h_{1}, h_{2}}$. Set

$$
\begin{aligned}
& \check{\theta}_{h_{1}, h_{2}}^{i}:=\theta_{h_{1}, h_{2}}^{i}-h_{1}^{2} \eta_{h_{1}, h_{2}}^{i 1}-h_{2}^{2} \eta_{h_{1}, h_{2}}^{i 2} \text { and } \\
& \check{\rho}_{h_{1}, h_{2}}^{i}:=\rho_{h_{1}, h_{2}}^{i}-h_{1}^{2} \xi_{h_{1}, h_{2}}^{i 1}-h_{2}^{2} \xi_{h_{1}, h_{2}}^{i 2}, \quad i=1,2 .
\end{aligned}
$$

Thus, we have from (78)-(89)

(90) $a\left(\check{\theta}_{h_{1}, h_{2}}^{1}, \boldsymbol{v}\right)-\left(\check{\rho}_{h_{1}, h_{2}}^{1}, \operatorname{div} \boldsymbol{v}\right)=O\left(h^{4}\right)\|\boldsymbol{v}\|_{0}, \quad \forall \boldsymbol{v} \in \boldsymbol{V}_{0, h_{1}, h_{2}}$,

(91) $\left(\operatorname{div} \check{\theta}_{h_{1}, h_{2}}^{1}, w\right)+\left(c \check{\rho}_{h_{1}, h_{2}}^{1}, w\right)+\left(\check{\rho}_{h_{1}, h_{2}}^{2}, w\right)=O\left(h^{4}\right)\|w\|_{0}, \quad \forall w \in W_{h_{1}, h_{2}}$,

(92) $a\left(\check{\theta}_{h_{1}, h_{2}}^{2}, \boldsymbol{v}\right)-\left(\check{\rho}_{h_{1}, h_{2}}^{2}, \operatorname{div} \boldsymbol{v}\right)=O\left(h^{4}\right)\|\boldsymbol{v}\|_{0}, \quad \forall \boldsymbol{v} \in \boldsymbol{V}_{0, h_{1}, h_{2}}$,

(93) $\left(\operatorname{div} \check{\theta}_{h_{1}, h_{2}}^{2}, w\right)+\left(c \check{\rho}_{h_{1}, h_{2}}^{2}, w\right)-\left(\check{\rho}_{h_{1}, h_{2}}^{1}, w\right)=O\left(h^{4}\right)\|w\|_{0}, \quad \forall w \in W_{h_{1}, h_{2}}$.

Following the steps for the estimates in the proof Theorem 3.1 yields by means of (90)-(93) that

$$
\left\|\check{\theta}_{h_{1}, h_{2}}^{1}\right\|_{\mathbf{v}}+\left\|\check{\theta}_{h_{1}, h_{2}}^{2}\right\| \mathbf{v}+\left\|\check{\rho}_{h_{1}, h_{2}}^{1}\right\|_{0}+\left\|\check{\rho}_{h_{1}, h_{2}}^{2}\right\|_{0} \leq C h^{4} .
$$

By the same argument as that for Theorem 3.3, we can establish the following result.

Theorem 3.6. We have under the conditions of Theorem 3.5 that

$$
\begin{aligned}
& P_{4 h_{1}, 4 h_{2}}^{3} y_{h_{1}, h_{2}}-y=h_{1}^{2} \xi^{11}+h_{2}^{2} \xi^{12}+\tilde{r}_{h_{1}, h_{2}}^{1}, \quad\left\|\tilde{r}_{h_{1}, h_{2}}^{1}\right\|_{0} \leq C h^{4}, \\
& P_{4 h_{1}, 4 h_{2}}^{3} z_{h_{1}, h_{2}}-z=h_{1}^{2} \xi^{21}+h_{2}^{2} \xi^{22}+\tilde{r}_{h_{1}, h_{2}}^{2}, \quad\left\|\tilde{r}_{h_{1}, h_{2}}^{2}\right\|_{0} \leq C h^{4}, \\
& \Pi_{4 h_{1}, 4 h_{2}}^{3} \boldsymbol{p}_{h_{1}, h_{2}}-\boldsymbol{p}=h_{1}^{2} \eta^{11}+h_{2}^{2} \eta^{12}+\tilde{\boldsymbol{r}}_{h_{1}, h_{2}}^{1}, \quad\left\|\tilde{\boldsymbol{r}}_{h_{1}, h_{2}}^{1}\right\|_{0} \leq C h^{4}, \\
& \Pi_{4 h_{1}, 4 h_{2}}^{3} \boldsymbol{q}_{h_{1}, h_{2}}-\boldsymbol{q}=h_{1}^{2} \eta^{21}+h_{2}^{2} \eta^{22}+\tilde{\boldsymbol{r}}_{h_{1}, h_{2}}^{2}, \quad\left\|\tilde{\boldsymbol{r}}_{h_{1}, h_{2}}^{2}\right\|_{0} \leq C h^{4},
\end{aligned}
$$

where $\left(\xi^{11}, \eta^{11}, \xi^{21}, \eta^{21}\right),\left(\xi^{12}, \eta^{12}, \xi^{22}, \eta^{22}\right) \in\left(W \times V_{0}\right)^{2}$ are the variational solutions of (78)-(81) and (82)-(85), respectively. 
From Theorem 3.6 one can obtain the following unidirectional Richardson extrapolation results under the $L^{2}$-norm:

$$
\begin{aligned}
& \frac{4\left(P_{2 h_{1}, 4 h_{2}}^{3} y_{h_{1} / 2, h_{2}}+P_{4 h_{1}, 2 h_{2}}^{3} y_{h_{1}, h_{2} / 2}\right)-5 P_{4 h_{1}, 4 h_{2}}^{3} y_{h_{1}, h_{2}}}{3}=y+O\left(h^{4}\right), \\
& \frac{4\left(P_{2 h_{1}, 4 h_{2}}^{3} z_{h_{1} / 2, h_{2}}+P_{4 h_{1}, 2 h_{2}}^{3} z_{h_{1}, h_{2} / 2}\right)-5 P_{4 h_{1}, 4 h_{2}}^{3} z_{h_{1}, h_{2}}}{3}=z+O\left(h^{4}\right), \\
& \frac{4\left(\Pi_{2 h_{1}, 4 h_{2}}^{3} \boldsymbol{p}_{h_{1} / 2, h_{2}}+\Pi_{4 h_{1}, 2 h_{2}}^{3} \boldsymbol{p}_{h_{1}, h_{2} / 2}\right)-5 \Pi_{4 h_{1}, 4 h_{2}}^{3} \boldsymbol{p}_{h_{1}, h_{2}}}{3}=\boldsymbol{p}+O\left(h^{4}\right), \\
& \frac{4\left(\Pi_{2 h_{1}, 4 h_{2}}^{3} \boldsymbol{q}_{h_{1} / 2, h_{2}}+\Pi_{4 h_{1}, 2 h_{2}}^{3} \boldsymbol{q}_{h_{1}, h_{2} / 2}\right)-5 \Pi_{4 h_{1}, 4 h_{2}}^{3} \boldsymbol{q}_{h_{1}, h_{2}}}{3}=\boldsymbol{q}+O\left(h^{4}\right),
\end{aligned}
$$

where $\left(y_{h_{1} / 2, h_{2}}, \boldsymbol{p}_{h_{1} / 2, h_{2}}, z_{h_{1} / 2, h_{2}}, \boldsymbol{q}_{h_{1} / 2, h_{2}}\right),\left(y_{h_{1}, h_{2} / 2}, \boldsymbol{p}_{h_{1}, h_{2} / 2}, z_{h_{1}, h_{2} / 2}, \boldsymbol{q}_{h_{1}, h_{2} / 2}\right)$, and $\left(y_{h_{1}, h_{2}}, \boldsymbol{p}_{h_{1}, h_{2}}, z_{h_{1}, h_{2}}, \boldsymbol{q}_{h_{1}, h_{2}}\right)$ are the mixed finite element solutions corresponding to the meshes $\mathcal{T}_{h_{1} / 2, h_{2}}, \mathcal{T}_{h_{1}, h_{2} / 2}$, and $\mathcal{T}_{h_{1}, h_{2}}$, respectively, and $\mathcal{T}_{h_{1} / 2, h_{2}}$ as well as $\mathcal{T}_{h_{1}, h_{2} / 2}$ are gained by subdividing each element of $\mathcal{T}_{h_{1}, h_{2}}$ into small congruent rectangles in the $x$-direction and $y$-direction, respectively.

Similar to (62)-(65), we can also construct a posteriori error estimators by virtue of (94)-(97).

Theorem 3.7. Under the assumptions of Theorem 3.6, we have

$$
\begin{aligned}
& \left\|y-P_{2 h_{1}, 4 h_{2}}^{3} y_{h_{1} / 2, h_{2}}\right\|_{0} \\
= & \frac{1}{3}\left\|P_{2 h_{1}, 4 h_{2}}^{3} y_{h_{1} / 2, h_{2}}+4 P_{4 h_{1}, 2 h_{2}}^{3} y_{h_{1}, h_{2} / 2}-5 P_{4 h_{1}, 4 h_{2}}^{3} y_{h_{1}, h_{2}}\right\|_{0}+O\left(h^{4}\right) \\
& \left\|z-P_{2 h_{1}, 4 h_{2}}^{3} z_{h_{1} / 2, h_{2}}\right\|_{0} \\
= & \frac{1}{3}\left\|P_{2 h_{1}, 4 h_{2}}^{3} z_{h_{1} / 2, h_{2}}+4 P_{4 h_{1}, 2 h_{2}}^{3} z_{h_{1}, h_{2} / 2}-5 P_{4 h_{1}, 4 h_{2}}^{3} z_{h_{1}, h_{2}}\right\|_{0}+O\left(h^{4}\right) \\
& \left\|\boldsymbol{p}-\Pi_{2 h_{1}, 4 h_{2}}^{3} \boldsymbol{p}_{h_{1} / 2, h_{2}}\right\|_{0} \\
= & \frac{1}{3}\left\|\Pi_{2 h_{1}, 4 h_{2}}^{3} \boldsymbol{p}_{h_{1} / 2, h_{2}}+4 \Pi_{4 h_{1}, 2 h_{2}}^{3} \boldsymbol{p}_{h_{1}, h_{2} / 2}-5 \Pi_{4 h_{1}, 4 h_{2}}^{3} \boldsymbol{p}_{h_{1}, h_{2}}\right\|_{0}+O\left(h^{4}\right) \\
& \left\|\boldsymbol{q}-\Pi_{2 h_{1}, 4 h_{2}}^{3} \boldsymbol{q}_{h_{1} / 2, h_{2}}\right\|_{0} \\
= & \frac{1}{3}\left\|\Pi_{2 h_{1}, 4 h_{2}}^{3} \boldsymbol{q}_{h_{1} / 2, h_{2}}+4 \Pi_{4 h_{1}, 2 h_{2}}^{3} \boldsymbol{q}_{h_{1}, h_{2} / 2}-5 \Pi_{4 h_{1}, 4 h_{2}}^{3} \boldsymbol{q}_{h_{1}, h_{2}}\right\|_{0}+O\left(h^{4}\right), \\
& \left\|y-P_{4 h_{1}, 2 h_{2}}^{3} y_{h_{1}, h_{2} / 2}\right\|_{0} \\
= & \frac{1}{3}\left\|P_{4 h_{1}, 2 h_{2}}^{3} y_{h_{1}, h_{2} / 2}+4 P_{2 h_{1}, 4 h_{2}}^{3} y_{h_{1} / 2, h_{2}}-5 P_{4 h_{1}, 4 h_{2}}^{3} y_{h_{1}, h_{2}}\right\|_{0}+O\left(h^{4}\right), \\
& \left\|z-P_{4 h_{1}, 2 h_{2}}^{3} z_{h_{1}, h_{2} / 2}\right\|_{0} \\
= & \frac{1}{3}\left\|P_{4 h_{1}, 2 h_{2}}^{3} z_{h_{1}, h_{2} / 2}+4 P_{2 h_{1}, 4 h_{2}}^{3} z_{h_{1} / 2, h_{2}}-5 P_{4 h_{1}, 4 h_{2}}^{3} z_{h_{1}, h_{2}}\right\|_{0}+O\left(h^{4}\right), \\
& \left\|\boldsymbol{p}-\Pi_{4 h_{1}, 2 h_{2}}^{3} \boldsymbol{p}_{h_{1}, h_{2} / 2}\right\|_{0} \\
= & \frac{1}{3}\left\|\Pi_{4 h_{1}, 2 h_{2}}^{3} \boldsymbol{p}_{h_{1}, h_{2} / 2}+4 \Pi_{2 h_{1}, 4 h_{2}}^{3} \boldsymbol{p}_{h_{1} / 2, h_{2}}-5 \Pi_{4 h_{1}, 4 h_{2}}^{3} \boldsymbol{p}_{h_{1}, h_{2}}\right\|_{0}+O\left(h^{4}\right),
\end{aligned}
$$




$$
\begin{aligned}
& \left\|\boldsymbol{q}-\Pi_{4 h_{1}, 2 h_{2}}^{3} \boldsymbol{q}_{h_{1}, h_{2} / 2}\right\|_{0} \\
= & \frac{1}{3}\left\|\Pi_{4 h_{1}, 2 h_{2}}^{3} \boldsymbol{q}_{h_{1}, h_{2} / 2}+4 \Pi_{2 h_{1}, 4 h_{2}}^{3} \boldsymbol{q}_{h_{1} / 2, h_{2}}-5 \Pi_{4 h_{1}, 4 h_{2}}^{3} \boldsymbol{q}_{h_{1}, h_{2}}\right\|_{0}+O\left(h^{4}\right) .
\end{aligned}
$$

Moreover, if there exist positive constants $C_{1}, C_{2}, \ldots, C_{8}$ and $\epsilon_{1}, \epsilon_{2}, \ldots, \epsilon_{8} \in$ $(0,1)$ such that

$$
\begin{aligned}
& \left\|y-P_{2 h_{1}, 4 h_{2}}^{3} y_{h_{1} / 2, h_{2}}\right\|_{0} \geq C_{1} h^{4-\epsilon_{1}}, \\
& \left\|z-P_{2 h_{1}, 4 h_{2}}^{3} z_{h_{1} / 2, h_{2}}\right\|_{0} \geq C_{2} h^{4-\epsilon_{2}}, \\
& \left\|\boldsymbol{p}-\Pi_{2 h_{1}, 4 h_{2}}^{3} \boldsymbol{p}_{h_{1} / 2, h_{2}}\right\|_{0} \geq C_{3} h^{4-\epsilon_{3}}, \\
& \left\|\boldsymbol{q}-P_{2 h_{1}, 4 h_{2}}^{3} \boldsymbol{q}_{h_{1} / 2, h_{2}}\right\|_{0} \geq C_{4} h^{4-\epsilon_{4}}, \\
& \left\|y-P_{4 h_{1}, 2 h_{2}}^{3} y_{h_{1}, h_{2} / 2}\right\|_{0} \geq C_{5} h^{4-\epsilon_{5}}, \\
& \left\|z-P_{4 h_{1}, 2 h_{2}}^{3} z_{h_{1}, h_{2} / 2}\right\|_{0} \geq C_{6} h^{4-\epsilon_{6}}, \\
& \left\|\boldsymbol{p}-\Pi_{4 h_{1}, 2 h_{2}}^{3} \boldsymbol{p}_{h_{1}, h_{2} / 2}\right\|_{0} \geq C_{7} h^{4-\epsilon_{7}}, \\
& \left\|\boldsymbol{q}-P_{4 h_{1}, 2 h_{2}}^{3} \boldsymbol{q}_{h_{1}, h_{2} / 2}\right\|_{0} \geq C_{8} h^{4-\epsilon_{8}},
\end{aligned}
$$

then we have

$$
\begin{aligned}
& \lim _{h \rightarrow 0} \frac{3\left\|y-P_{2 h_{1}, 4 h_{2}}^{3} y_{h_{1} / 2, h_{2}}\right\|_{0}}{\left\|P_{2 h_{1}, 4 h_{2}}^{3} y_{h_{1} / 2, h_{2}}+4 P_{4 h_{1}, 2 h_{2}}^{3} y_{h_{1}, h_{2} / 2}-5 P_{4 h_{1}, 4 h_{2}}^{3} y_{h_{1}, h_{2}}\right\|_{0}}=1, \\
& \lim _{h \rightarrow 0} \frac{3\left\|z-P_{2 h_{1}, 4 h_{2}}^{3} z_{h_{1} / 2, h_{2}}\right\|_{0}}{\left\|P_{2 h_{1}, 4 h_{2}}^{3} z_{h_{1} / 2, h_{2}}+4 P_{4 h_{1}, 2 h_{2}}^{3} z_{h_{1}, h_{2} / 2}-5 P_{4 h_{1}, 4 h_{2}}^{3} z_{h_{1}, h_{2}}\right\|_{0}}=1, \\
& \lim _{h \rightarrow 0} \frac{3\left\|\boldsymbol{p}-\Pi_{2 h_{1}, 4 h_{2}}^{3} \boldsymbol{p}_{h_{1} / 2, h_{2}}\right\|_{0}}{\left\|\Pi_{2 h_{1}, 4 h_{2}}^{3} \boldsymbol{p}_{h_{1} / 2, h_{2}}+4 \Pi_{4 h_{1}, 2 h_{2}}^{3} \boldsymbol{p}_{h_{1}, h_{2} / 2}-5 \Pi_{4 h_{1}, 4 h_{2}}^{3} \boldsymbol{p}_{h_{1}, h_{2}}\right\|_{0}}=1 \text {, } \\
& \lim _{h \rightarrow 0} \frac{3\left\|\boldsymbol{q}-\Pi_{2 h_{1}, 4 h_{2}}^{3} \boldsymbol{q}_{h_{1} / 2, h_{2}}\right\|_{0}}{\left\|\Pi_{2 h_{1}, 4 h_{2}}^{3} \boldsymbol{q}_{h_{1} / 2, h_{2}}+4 \Pi_{4 h_{1}, 2 h_{2}}^{3} \boldsymbol{q}_{h_{1}, h_{2} / 2}-5 \Pi_{4 h_{1}, 4 h_{2}}^{3} \boldsymbol{q}_{h_{1}, h_{2}}\right\|_{0}}=1 \text {, } \\
& \lim _{h \rightarrow 0} \frac{3\left\|y-P_{4 h_{1}, 2 h_{2}}^{3} y_{h_{1}, h_{2} / 2}\right\|_{0}}{\left\|P_{4 h_{1}, 2 h_{2}}^{3} y_{h_{1}, h_{2} / 2}+4 P_{2 h_{1}, 4 h_{2}}^{3} y_{h_{1} / 2, h_{2}}-5 P_{4 h_{1}, 4 h_{2}}^{3} y_{h_{1}, h_{2}}\right\|_{0}}=1, \\
& \lim _{h \rightarrow 0} \frac{3\left\|z-P_{4 h_{1}, 2 h_{2}}^{3} z_{h_{1}, h_{2} / 2}\right\|_{0}}{\left\|P_{4 h_{1}, 2 h_{2}}^{3} z_{h_{1}, h_{2} / 2}+4 P_{2 h_{1}, 4 h_{2}}^{3} z_{h_{1} / 2, h_{2}}-5 P_{4 h_{1}, 4 h_{2}}^{3} z_{h_{1}, h_{2}}\right\|_{0}}=1, \\
& \lim _{h \rightarrow 0} \frac{3\left\|\boldsymbol{p}-\Pi_{4 h_{1}, 2 h_{2}}^{3} \boldsymbol{p}_{h_{1}, h_{2} / 2}\right\|_{0}}{\left\|\Pi_{4 h_{1}, 2 h_{2}}^{3} \boldsymbol{p}_{h_{1}, h_{2} / 2}+4 \Pi_{2 h_{1}, 4 h_{2}}^{3} \boldsymbol{p}_{h_{1} / 2, h_{2}}-5 \Pi_{4 h_{1}, 4 h_{2}}^{3} \boldsymbol{p}_{h_{1}, h_{2}}\right\|_{0}}=1 \text {, } \\
& \lim _{h \rightarrow 0} \frac{3\left\|\boldsymbol{q}-\Pi_{4 h_{1}, 2 h_{2}}^{3} \boldsymbol{q}_{h_{1}, h_{2} / 2}\right\|_{0}}{\left\|\Pi_{4 h_{1}, 2 h_{2}}^{3} \boldsymbol{q}_{h_{1}, h_{2} / 2}+4 \Pi_{2 h_{1}, 4 h_{2}}^{3} \boldsymbol{q}_{h_{1} / 2, h_{2}}-5 \Pi_{4 h_{1}, 4 h_{2}}^{3} \boldsymbol{q}_{h_{1}, h_{2}}\right\|_{0}}=1 .
\end{aligned}
$$

\section{The interpolation defect correction}

In this section we propose and investigate an interpolation defect correction scheme (see, for example, [6, 23]) applied to the mixed finite element solution $\left(y_{h_{1}, h_{2}}, \boldsymbol{p}_{h_{1}, h_{2}}, z_{h_{1}, h_{2}}, \boldsymbol{q}_{h_{1}, h_{2}}\right) \in\left(W_{h_{1}, h_{2}} \times \boldsymbol{V}_{0, h_{1}, h_{2}}\right)^{2}$ to obtain approximations 
with higher convergence rate. Also, these new approximations are naturally used to form a posteriori error estimators in order to estimate the actual accuracy of the mixed finite element solutions.

First of all, for the future need we construct two projection interpolation operators $\Pi_{2 h_{1}, 2 h_{2}}^{1}$ and $P_{2 h_{1}, 2 h_{2}}^{1}$ associated with $\mathcal{T}_{2 h_{1}, 2 h_{2}}$ satisfy

$$
\begin{aligned}
& \Pi_{2 h_{1}, 2 h_{2}}^{1} \Pi_{h_{1}, h_{2}}^{0}=\Pi_{2 h_{1}, 2 h_{2}}^{1}, \\
& \left\|\Pi_{2 h_{1}, 2 h_{2}}^{1} \boldsymbol{v}\right\|_{0} \leq C\|\boldsymbol{v}\|_{0}, \quad \forall \boldsymbol{v} \in \boldsymbol{V}_{0, h_{1}, h_{2}}, \\
& \left\|\Pi_{2 h_{1}, 2 h_{2}}^{1} \mathbf{u}-\mathbf{u}\right\|_{0} \leq C h^{2}\|\mathbf{u}\|_{2}, \quad \forall \mathbf{u} \in\left(H^{2}(\Omega)\right)^{2}, \\
& P_{2 h_{1}, 2 h_{2}}^{1} P_{h_{1}, h_{2}}^{0}=P_{2 h_{1}, 2 h_{2}}^{1}, \\
& \left\|P_{2 h_{1}, 2 h_{2}}^{1} w\right\|_{0} \leq C\|w\|_{0}, \quad \forall w \in W_{h_{1}, h_{2}}, \\
& \left\|P_{2 h_{1}, 2 h_{2}}^{1} p-p\right\|_{0} \leq C h^{2}\|p\|_{2}, \quad \forall p \in H^{2}(\Omega) .
\end{aligned}
$$

Then, like that seen in the last section, it is assumed that the rectangular partition $\mathcal{T}_{h_{1}, h_{2}}$ has been obtained from $\mathcal{T}_{2 h_{1}, 2 h_{2}}$ with mesh size $2 h$ by subdividing each element of $\mathcal{T}_{2 h_{1}, 2 h_{2}}$ into four small congruent rectangles. Let $\hat{e}:=\bigcup_{i=1}^{4} e_{i}$ with $e_{i} \in \mathcal{T}_{h_{1}, h_{2}}$. And this, the two interpolation operators $\Pi_{2 h_{1}, 2 h_{2}}^{1}$ and $P_{2 h_{1}, 2 h_{2}}^{1}$ associated with $\mathcal{T}_{2 h_{1}, 2 h_{2}}$ of degree at most 1 in $x$ and $y$ on $\hat{e}$, respectively, and defined as follows:

$$
\begin{aligned}
& \left.\Pi_{2 h_{1}, 2 h_{2}}^{1} \mathbf{u}\right|_{\hat{e}} \in Q_{2,1}(\hat{e}) \times Q_{1,2}(\hat{e}),\left.\quad P_{2 h_{1}, 2 h_{2}}^{1} p\right|_{\hat{e}} \in Q_{1,1}(\hat{e}), \\
& \int_{s_{i}}\left(\mathbf{u}-\Pi_{2 h_{1}, 2 h_{2}}^{1} \mathbf{u}\right) \cdot \boldsymbol{n} d s=0, \quad i=1,2, \ldots, 12, \\
& \int_{e_{i}}\left(p-P_{2 h_{1}, 2 h_{2}}^{1} p\right)=0, \quad i=1,2,3,4
\end{aligned}
$$

where $s_{i}(i=1,2, \ldots, 12)$ is one of the twelve sides of the four small elements $e_{i}(i=1,2,3,4)$. We can also check that the two operators $\Pi_{2 h_{1}, 2 h_{2}}^{1}$ and $P_{2 h_{1}, 2 h_{2}}^{1}$ defined above satisfy the properties indicated in (98)-(103).

In addition, we also need two pairs of mixed finite element projection operators $R_{h_{1}, h_{2}}^{1} \times S_{h_{1}, h_{2}}^{1} \times R_{h_{1}, h_{2}}^{2} \times S_{h_{1}, h_{2}}^{2}:\left(W \times \boldsymbol{V}_{0}\right)^{2} \rightarrow\left(W_{h_{1}, h_{2}} \times V_{0, h_{1}, h_{2}}\right)^{2}$ defined by

$$
\begin{aligned}
& a\left(S_{h_{1}, h_{2}}^{1} \boldsymbol{p}-\boldsymbol{p}, \boldsymbol{v}\right)-\left(R_{h_{1}, h_{2}}^{1} y-y, \operatorname{div} \boldsymbol{v}\right)=0, \\
& \left(\operatorname{div}\left(S_{h_{1}, h_{2}}^{1} \boldsymbol{p}-\boldsymbol{p}\right), w\right)+\left(c\left(R_{h_{1}, h_{2}}^{1} y-y\right), w\right)+\left(R_{h_{1}, h_{2}}^{2} z-z, w\right)=0, \\
& a\left(S_{h_{1}, h_{2}}^{2} \boldsymbol{q}-\boldsymbol{q}, \boldsymbol{v}\right)-\left(R_{h_{1}, h_{2}}^{2} z-z, \operatorname{div} \boldsymbol{v}\right)=0, \\
& \left(\operatorname{div}\left(S_{h_{1}, h_{2}}^{2} \boldsymbol{q}-\boldsymbol{q}\right), w\right)+\left(c\left(R_{h_{1}, h_{2}}^{2} z-z\right), w\right)-\left(R_{h_{1}, h_{2}}^{1} y-y, w\right)=0
\end{aligned}
$$

for all $\boldsymbol{v} \in \boldsymbol{V}_{0, h_{1}, h_{2}}$ and $\boldsymbol{w} \in W_{h_{1}, h_{2}}$. Then, $\left(R_{h_{1}, h_{2}}^{1} y, S_{h_{1}, h_{2}}^{1} \boldsymbol{p}, R_{h_{1}, h_{2}}^{2} z, S_{h_{1}, h_{2}}^{2} \boldsymbol{q}\right)$ is the solution of (20)-(23) if (y, p, z, q) is the solution of (14)-(17).

Theorem 4.1. Suppose that the conditions of Theorem 3.3 are fulfilled. Then, we have

(104) $\left\|y_{h_{1}, h_{2}}^{*}-y\right\|_{0}+\left\|z_{h_{1}, h_{2}}^{*}-z\right\|_{0}+\left\|\boldsymbol{p}_{h_{1}, h_{2}}^{*}-\boldsymbol{p}\right\|_{0}+\left\|\boldsymbol{q}_{h_{1}, h_{2}}^{*}-\boldsymbol{q}\right\|_{0} \leq C h^{4}$, 
where

$$
\begin{aligned}
& y_{h_{1}, h_{2}}^{*}:=P_{4 h_{1}, 4 h_{2}}^{3} y_{h_{1}, h_{2}}+P_{2 h_{1}, 2 h_{2}}^{1} y_{h_{1}, h_{2}}-P_{2 h_{1}, 2 h_{2}}^{1} R_{h_{1}, h_{2}}^{1} P_{4 h_{1}, 4 h_{2}}^{3} y_{h_{1}, h_{2}}, \\
& z_{h_{1}, h_{2}}^{*}:=P_{4 h_{1}, 4 h_{2}}^{3} z_{h_{1}, h_{2}}+P_{2 h_{1}, 2 h_{2}}^{1} z_{h_{1}, h_{2}}-P_{2 h_{1}, 2 h_{2}}^{1} R_{h_{1}, h_{2}}^{2} P_{4 h_{1}, 4 h_{2}}^{3} z_{h_{1}, h_{2}}, \\
& \boldsymbol{p}_{h_{1}, h_{2}}^{*}:=\Pi_{4 h_{1}, 4 h_{2}}^{3} \boldsymbol{p}_{h_{1}, h_{2}}+\Pi_{2 h_{1}, 2 h_{2}}^{1} \boldsymbol{p}_{h_{1}, h_{2}}-\Pi_{2 h_{1}, 2 h_{2}}^{1} S_{h_{1}, h_{2}}^{1} \Pi_{4 h_{1}, 4 h_{2}}^{3} \boldsymbol{p}_{h_{1}, h_{2}}, \\
& \boldsymbol{q}_{h_{1}, h_{2}}^{*}:=\Pi_{4 h_{1}, 4 h_{2}}^{3} \boldsymbol{q}_{h_{1}, h_{2}}+\Pi_{2 h_{1}, 2 h_{2}}^{1} \boldsymbol{q}_{h_{1}, h_{2}}-\Pi_{2 h_{1}, 2 h_{2}}^{1} S_{h_{1}, h_{2}}^{2} \Pi_{4 h_{1}, 4 h_{2}}^{3} \boldsymbol{q}_{h_{1}, h_{2}} .
\end{aligned}
$$

Proof. It has been proved in Theorem 3.3 that

$$
P_{4 h_{1}, 4 h_{2}}^{3} y_{h_{1}, h_{2}}-y=h^{2} \xi^{1}+\gamma_{h_{1}, h_{2}}^{1 *}, \quad\left\|\gamma_{h_{1}, h_{2}}^{1 *}\right\|_{0} \leq C h^{4} .
$$

Then, multiplying this equality by the operator $\left(I-P_{2 h_{1}, 2 h_{2}}^{1} R_{h_{1}, h_{2}}^{1}\right)$, where $I$ is the identity operator, results in

$$
\begin{aligned}
& \left(I-P_{2 h_{1}, 2 h_{2}}^{1} R_{h_{1}, h_{2}}^{1}\right)\left(P_{4 h_{1}, 4 h_{2}}^{3} y_{h_{1}, h_{2}}-y\right) \\
= & h^{2}\left(I-P_{2 h_{1}, 2 h_{2}}^{1} R_{h_{1}, h_{2}}^{1}\right) \xi^{1}+O\left(h^{4}\right) \\
= & h^{2}\left(\xi^{1}-P_{2 h_{1}, 2 h_{2}}^{1} \xi^{1}\right)+h^{2}\left(P_{2 h_{1}, 2 h_{2}}^{1} \xi^{1}-P_{2 h_{1}, 2 h_{2}}^{1} \xi_{h_{1}, h_{2}}^{1}\right)+O\left(h^{4}\right) \\
= & h^{2} P_{2 h_{1}, 2 h_{2}}^{1}\left(P_{h_{1}, h_{2}}^{0} \xi^{1}-\xi_{h_{1}, h_{2}}^{1}\right)+O\left(h^{4}\right),
\end{aligned}
$$

where we used

$$
\left\|\xi^{1}-P_{2 h_{1}, 2 h_{2}}^{1} \xi^{1}\right\|_{0} \leq C h^{2}\left\|\xi^{1}\right\|_{2} \text { and } P_{2 h_{1}, 2 h_{2}}^{1} P_{h_{1}, h_{2}}^{0}=P_{2 h_{1}, 2 h_{2}}^{1}
$$

according to the properties of the operator $P_{2 h_{1}, 2 h_{2}}^{1}$ described in (101)-(103). Furthermore, it follows from Lemma 3.2 and the inequality

$$
\left\|P_{2 h_{1}, 2 h_{2}}^{1}\left(P_{h_{1}, h_{2}}^{0} \xi^{1}-\xi_{h_{1}, h_{2}}^{1}\right)\right\|_{0} \leq C\left\|P_{h_{1}, h_{2}}^{0} \xi^{1}-\xi_{h_{1}, h_{2}}^{1}\right\|_{0}
$$

that

$$
\left(I-P_{2 h_{1}, 2 h_{2}}^{1} R_{h_{1}, h_{2}}^{1}\right)\left(P_{4 h_{1}, 4 h_{2}}^{3} y_{h_{1}, h_{2}}-y\right)=O\left(h^{4}\right),
$$

and the left-hand side is nothing but

$$
\left(I-P_{2 h_{1}, 2 h_{2}}^{1} R_{h_{1}, h_{2}}^{1}\right)\left(P_{4 h_{1}, 4 h_{2}}^{3} y_{h_{1}, h_{2}}-y\right)=y_{h_{1}, h_{2}}^{*}-y .
$$

Similarly, we can gain other terms of (104).

Analogous to Section 3 we can utilize the superconvergent approximation provided in Theorem 4.1 to establish a posteriori error estimators for the mixed finite element solution of the problem (14)-(17). In fact, we have:

Theorem 4.2. If the conditions of Theorem 4.1 are satisfied, then we have

$$
\begin{aligned}
& \left\|y-y_{h_{1}, h_{2}}\right\|_{0}=\left\|y_{h_{1}, h_{2}}^{*}-y_{h_{1}, h_{2}}\right\|_{0}+O\left(h^{4}\right), \\
& \left\|z-z_{h_{1}, h_{2}}\right\|_{0}=\left\|z_{h_{1}, h_{2}}^{*}-z_{h_{1}, h_{2}}\right\|_{0}+O\left(h^{4}\right), \\
& \left\|\boldsymbol{p}-\boldsymbol{p}_{h_{1}, h_{2}}\right\|_{0}=\left\|\boldsymbol{p}_{h_{1}, h_{2}}^{*}-\boldsymbol{p}_{h_{1}, h_{2}}\right\|_{0}+O\left(h^{4}\right), \\
& \left\|\boldsymbol{q}-\boldsymbol{q}_{h_{1}, h_{2}}\right\|_{0}=\left\|\boldsymbol{q}_{h_{1}, h_{2}}^{*}-\boldsymbol{q}_{h_{1}, h_{2}}\right\|_{0}+O\left(h^{4}\right) .
\end{aligned}
$$

Furthermore, if there exist positive constants $C_{1}, C_{2}, C_{3}, C_{4}$ and sufficiently small $\epsilon_{1}, \epsilon_{2}, \epsilon_{3}, \epsilon_{4} \in(0,1)$ such that

$$
\left\|y-y_{h_{1}, h_{2}}\right\|_{0} \geq C_{1} h^{4-\epsilon_{1}}, \quad\left\|z-z_{h_{1}, h_{2}}\right\|_{0} \geq C_{2} h^{4-\epsilon_{2}},
$$




$$
\left\|\boldsymbol{p}-\boldsymbol{p}_{h_{1}, h_{2}}\right\|_{0} \geq C_{3} h^{4-\epsilon_{3}}, \quad\left\|\boldsymbol{q}-\boldsymbol{q}_{h_{1}, h_{2}}\right\|_{0} \geq C_{4} h^{4-\epsilon_{4}},
$$

then there hold

$$
\begin{array}{ll}
\lim _{h \rightarrow 0} \frac{\left\|y-y_{h_{1}, h_{2}}\right\|_{0}}{\left\|y_{h_{1}, h_{2}}^{*}-y_{h_{1}, h_{2}}\right\|_{0}}=1, & \lim _{h \rightarrow 0} \frac{\left\|z-z_{h_{1}, h_{2}}\right\|_{0}}{\left\|z_{h_{1}, h_{2}}^{*}-z_{h_{1}, h_{2}}\right\|_{0}}=1, \\
\lim _{h \rightarrow 0} \frac{\left\|\boldsymbol{p}-\boldsymbol{p}_{h_{1}, h_{2}}\right\|_{0}}{\left\|\boldsymbol{p}_{h_{1}, h_{2}}^{*}-\boldsymbol{p}_{h_{1}, h_{2}}\right\|_{0}}=1, & \lim _{h \rightarrow 0} \frac{\left\|\boldsymbol{q}-\boldsymbol{q}_{h_{1}, h_{2}}\right\|_{0}}{\left\|\boldsymbol{q}_{h_{1}, h_{2}}^{*}-\boldsymbol{q}_{h_{1}, h_{2}}\right\|_{0}}=1 .
\end{array}
$$

\section{Conclusion and future works}

In this paper, we derived asymptotic error expansions in the sense of $L^{2}$ norm for the mixed finite element approximation to a class of optimal control problems under rectangular meshes. Based on the asymptotic error expansions, the Richardson extrapolation of two different schemes and an interpolation defect correction are given. Furthermore, as a result of all these higher order numerical approximations, they can be used to generate a posteriori error estimators for the mixed finite element approximation. It should be pointed out that the assumption of the high regularity of the solutions to the state and adjoint equations is too strong for many practical problems. However, is still significant to provide these numerical schemes with high accuracy for optimal control problems in either theory or practice.

There are many important issues remaining to be addressed in this area, including high accuracy analysis in the sense of $L^{\infty}$-norm and for more complicated control problems. Moreover, many computational issues have to be addressed for designing high accurate numerical methods for the optimal control problems.

\section{References}

[1] W. Alt, On the approximation of infinite optimization problems with an application to optimal control problems, Appl. Math. Optim. 12 (1984), no. 1, 15-27.

$[2]$ W. Alt and U. Machenroth, Convergence of finite element approximations to state constrained convex parabolic boundary control problems, SIAM J. Control Optim. 27 (1989), no. $4,718-736$.

[3] N. Arada, E. Casas, and F. Tröltzsch, Error estimates for the numerical approximation of a semilinear elliptic control problem, Comput. Optim. Appl. 23 (2002), no. 2, 201229.

[4] H. Blum, Q. Lin, and R. Rannacher, Asymptotic error expansion and Richardson extrapolation for linear finite elements, Numer. Math. 49 (1986), no. 1, 11-37.

[5] H. Brunner, Y. Lin, and S. Zhang, Higher accuracy methods for second-kind Volterra integral equations based on asymptotic expansions of iterated Galerkin methods, J. Integral Equations Appl. 10 (1998), no. 4, 375-396.

[6] C. Chen and Y. Huang, Higher Accuracy Theory of FEM, Hunan Science Press, Changsha, China, 1995.

[7] Y. Chen, Superconvergence of mixed finite element methods for optimal control problems, Math. Comp. 77 (2008), no. 263, 1269-1291.

[8] _ Superconvergence of quadratic optimal control problems by triangular mixed finite elements, Internat. J. Numer. Methods Engrg. 75 (2008), no. 8, 881-898. 
[9] Y. Chen and W. B. Liu, Error estimates and superconvergence of mixed finite elements for quadratic optimal control, Int. J. Numer. Anal. Model. 3 (2006), no. 3, 311-321.

[10] _ A posteriori error estimates for mixed finite element solutions of convex optimal control problems, J. Comput. Appl. Math. 211 (2008), no. 1, 76-89.

[11] Y. Chen and Z. Lu, Error estimates of fully discrete mixed finite element methods for semilinear quadratic parabolic optimal control problem, Comput. Methods Appl. Mech. Engrg. 199 (2010), no. 23-24, 1415-1423.

[12] I. Chryssoverghi, Discretization methods for semilinear parabolic optimal control problems, Int. J. Numer. Anal. Model. 3 (2006), no. 4, 437-458.

[13] J. Douglas and J. E. Roberts, Global estimates for mixed methods for second order elliptic equations, Math. Comp. 44 (1985), no. 169, 39-52.

[14] R. E. Ewing, Y. Lin, T. Sun, J. Wang, and S. Zhang, Sharp L L-error estimates and superconvergence of mixed finite element methods for non-Fickian flows in porous media, SIAM J. Numer. Anal. 40 (2002), no. 4, 1538-1560.

[15] G. Fairweather, Q. Lin, Y. Lin, J. Wang, and S. Zhang, Asymptotic expansions and Richardson extrapolation of approximate solutions for second order elliptic problems on rectangular domains by mixed finite element methods, SIAM J. Numer. Anal. 44 (2006), no. 3, 1122-1149.

[16] M. D. Gunzburger and S. L. Hou, Finite-dimensional approximation of a class of constrained nonlinear optimal control problems, SIAM J. Control Optim. 34 (1996), no. 3, 1001-1043.

[17] J. Haslinger and P. Neittaanmaki, Finite Element Approximation for Optimal Shape Design, John Wiley and Sons, Chichester, UK, 1988.

[18] P. Helfrich, Asymptotic expansion for the finite element approximation of parabolic problems, Extrapolation procedures in the finite element method (Bonn, 1983), 11-30, Bonner Math. Schriften, 158, Univ. Bonn, Bonn, 1984.

[19] L. Hou and J. C. Turner, Analysis and finite element approximation of an optimal control problem in electrochemistry with current density controls, Numer. Math. 71 (1995), no. 3, 289-315.

[20] G. Knowles, Finite element approximation of parabolic time optimal control problems, SIAM J. Control Optim. 20 (1982), no. 3, 414-427.

[21] Q. Lin, I. H. Sloan, and R. Xie, Extrapolation of the iterated-collocation method for integral equations, SIAM J. Numer. Anal. 27 (1990), no. 6, 1535-1541.

[22] Q. Lin and N. Yan, The Construction and Analysis of High Efficiency Finite Element Methods, Hebei University Press, Baoding, China, 1996.

[23] Q. Lin, S. Zhang, and N. Yan, Extrapolation and defect correction for diffusion equations with boundary integral conditions, Acta Math. Sci. Ser. B Engl. Ed. 17 (1997), no. 4, 405-412.

[24] _ High accuracy analysis for integrodifferential equations, Acta Math. Appl. Sin. Engl. Ser. 14 (1998), no. 2, 202-211.

[25] _ Methods for improving approximate accuracy for hyperbolic integrodifferential equations, J. Systems Sci. Math. Sci. 10 (1997), no. 3, 282-288.

[26] T. Lin, Y. Lin, M. Rao, and S. Zhang, Petrov-Galerkin methods for linear Volterra integro-differential equations, SIAM J. Numer. Anal. 38 (2000), no. 3, 937-963.

[27] J. L. Lions, Optimal Control of Systems Governed by Partial Differential Equations, Springer, Berlin, 1971.

[28] H. Liu and N. Yan, Global Superconvergence for optimal control problems governed by Stokes equations, Int. J. Numer. Anal. Model. 3 (2006), no. 3, 283-302.

[29] T. Liu, N. Yan, and S. Zhang, Richardson extrapolation and defect correction of finite element methods for optimal control problems, J. Comput. Math. 28 (2010), no. 1, $55-71$. 
[30] R. S. Mcknight and W. E. Borsarge, The Ritz-Galerkin procedure for parabolic control problems, SIAM J. Control Optim. 11 (1973), 510-524.

[31] P. Neittaanmaki and D. Tiba, Optimal Control of Nonlinear Parabolic Systems: Theory, Algorithms and Applications, M. Dekker, New York, 1994.

[32] D. Tiba, Lectures on the Optimal Control of Elliptic Problems, University of Jyvaskyla Press, Jyvaskyla, Finland, 1995.

[33] F. Troltzsch, Semidiscrete Ritz-Galerkin approximation of nonlinear parabolic boundary control problems-strong convergence of optimal control, Appl. Math. Optim. 29 (1994), no. 3, 309-329.

[34] J. Wang, Superconvergence and extrapolation for mixed finite element methods on rectangular domains, Math. Comp. 56 (1991), no. 194, 477-503.

[35] _ Asymptotic expansions and $L^{\infty}$-error estimates for mixed finite element methods for second order elliptic problems, Numer. Math. 55 (1989), no. 4, 401-430.

[36] X. Xing and Y. Chen, Error estimates of mixed methods for optimal control problems governed by parabolic equations, Internat. J. Numer. Methods Engrg. 75 (2008), no. 6, $735-754$.

[37] N. Yan and K. Li, An extrapolation method for BEM, J. Comput. Math. 2 (1989), no. $2,217-224$.

[38] S. Zhang, T. Lin, Y. Lin, and M. Rao, Extrapolation and a-posteriori error estimators of Petrov-Galerkin methods for non-linear Volterra integro-differential equations, J. Comput. Math. 19 (2001), no. 4, 407-422.

YANPING CHEN

School of Mathematical Sciences

South China Normal University

Guangzhou 510631, P. R. China

E-mail address: yanpingchen@scnu.edu.cn

YUNQING HUANG

HunAN Key Laboratory FOR COMPUTATION

and Simulation in Science And Engineering

Department of Mathematics

XIANGTAN UNIVERSITY

Xiangtan 411105, P. R. China

E-mail address: huangyq@xtu.edu.cn

Tianliang Hou

Hunan Key Laboratory for Computation And Simulation in Science And Engineering Department of Mathematics

Xiangtan University

Xiangtan 411105, P. R. China

E-mail address: htlchb@163.com 